\title{
Der Mindestlohn in der Pflegebranche - Die Folgen eines Mindestlohns in einer Wachstumsbranche
}

\author{
Katrin Harsch • Hans Verbeek
}

Angenommen: 15. Oktober 2012 / Online publiziert: 5. Dezember 2012

(C) Institut für Arbeitsmarkt- und Berufsforschung 2012

Zusammenfassung Mit der Einführung eines Mindestlohns in der Pflegebranche im August 2010 wurde erstmals in einem Gesundheitssektor ein Mindestlohn eingeführt. Zudem ist die Pflegebranche im Unterschied zu anderen Branchen mit Mindestlöhnen in den letzten Jahren kontinuierlich gewachsen. Unsere Ergebnisse zeigen, dass insbesondere ostdeutsche Einrichtungen vom Mindestlohn betroffen waren, während in den meisten westdeutschen Einrichtungen die Löhne bereits vor Einführung deutlich höher waren. Daher können für Ostdeutschland Lohneffekte gefunden werden, während keine Auswirkungen auf die Beschäftigung identifiziert werden können.

Schlüsselwörter Mindestlohn · Beschäftigung · Löhne · Regulierung des Arbeitsmarkts

JEL Klassifikationen $\mathrm{J} 38 \cdot \mathrm{J} 20 \cdot \mathrm{I} 11 \cdot \mathrm{I} 18$

\section{The Minimum Wage in the Care Secor-The Consequences of a Minimum Wage in a Growth Sector}

\begin{abstract}
The introduction of a minimum wage in the care sector in August 2010 was the first time that a minimum wage was introduced in a health sector. Furthermore, in contrast to other industries with minimum wages, the care sector has continually increased in recent years. Our results
\end{abstract}

Für die in diesem Beitrag publizierten Ergebnisse und Schlussfolgerungen zeichnen ausschließlich die Autorinnen und Autoren verantwortlich. Sie geben nicht notwendigerweise die Position des Bundesministerium für Arbeit und Soziales wieder.

K. Harsch $\cdot$ H. Verbeek $(\bowtie)$

Institut für Angewandte Wirtschaftsforschung (IAW) e.V., Ob dem Himmelreich 1, 72074 Tübingen, Deutschland

e-mail: hans.verbeek@iaw.edu show that especially East German facilities were affected by the minimum wage, while most West German facilities paid significantly higher wages already prior to the introduction. Therefore, for East Germany wage effects can be found, whereas no effects on employment can be identified.

\section{Einleitung}

Die Pflegebranche unterscheidet sich deutlich von den anderen Branchen mit Mindestlöhnen nach dem Arbeitnehmerentsendegesetz (AEntG). Sie ist die einzige Branche im Gesundheitsbereich mit einem Mindestlohn. Zudem gehört sie mit 760.577 sozialversicherungspflichtig Beschäftigten zu den Branchen mit den meisten Erwerbstätigen und ist in den letzten zehn Jahren konstant gewachsen. Nach der Statistik der Bundesagentur für Arbeit (2012) umfasst die Pflegebranche damit etwa 2,6\% aller sozialversicherungspflichtigen Beschäftigten. Dies ist vergleichbar mit der Anzahl sozialversicherungspflichtig Beschäftigter in der Automobilindustrie (805.518). Unter den Branchen mit einem Mindestlohn arbeiten lediglich im Bauhauptgewerbe ähnlich viele Erwerbstätige wie in der Pflegebranche.

Aufgrund der demographischen Entwicklung ist mit einem weiter wachsenden Fachkräftebedarf in der Pflegebranche zu rechnen. Der zunehmende Fachkräftebedarf kann bereits seit 2005 nicht mehr durch Pflegefachkräfte gedeckt werden (Afentakis and Maier 2010). Gleichzeitig ist die Pflegebranche durch eine sehr heterogene Trägerlandschaft gekennzeichnet. Entsprechend uneinheitlich ist auch die Tariflandschaft strukturiert, da die großen freigemeinnützigen Träger eigenständige Tarifverträge haben und die kirchli- 
chen Trägerorganisationen keine Tarifvereinbarungen treffen, sondern Arbeitsbedingungen nach dem sogenannten Dritten Weg in Arbeitsrechtlichen Kommissionen (Lührs 2010) festlegen.

Die Ausgangslage in der Pflegebranche wirft demnach einige Fragen auf, die im Folgenden diskutiert werden sollen: Inwieweit werden die Besonderheiten der Branche bei der Umsetzung des Mindestlohns berücksichtigt? Welche Auswirkungen sind durch einen Mindestlohn in einer Wachstumsbranche mit zunehmendem Fachkräftemangel zu erwarten und empirisch festzustellen? Ist die Festsetzung eines Mindestlohns das geeignete Mittel, um die Probleme auf dem Arbeitsmarkt für Pflegeleistungen zu lösen?

Zur Beantwortung dieser Fragen werden zunächst die Rahmenbedingungen bei der Einführung des Mindestlohns kurz beschrieben. Anschließend werden die Methoden dargestellt, die im Rahmen dieser Studie verwendet werden. Es wird eine Kombination aus quantitativen und qualitativen Methoden gewählt. Dadurch ist eine umfassendere Bewertung der Auswirkungen des Mindestlohns möglich. Es wird erläutert, welche Methoden angewendet und wie die Erkenntnisse aus den Untersuchungsschritten miteinander verbunden werden. Aufgrund der erst kürzlich stattgefundenen Mindestlohneinführung wird bei der Beschreibung der Datenlage die Problematik fehlender amtlicher Daten für den Zeitraum nach der Mindestlohneinführung diskutiert und die für die Wirkungsanalysen verwendeten Befragungsdaten detailliert vorgestellt. In den deskriptiven Analysen werden sowohl die Entwicklung als auch die Situation in der Pflegebranche in den Jahren vor der Mindestlohneinführung beschrieben. Anschließend werden verschiedene Maße der Eingriffsintensität sowie die Lohnverteilung diskutiert. Zu Beginn des Ergebnisteils werden die Modellspezifikationen dargelegt. Die Ergebnisse der Evaluation werden differenziert nach Auswirkungen auf den Lohn, die Beschäftigung und den Wettbewerb. Zudem wird thematisiert, wie die vom Mindestlohn betroffenen Akteure diesen einschätzen und bewerten. Abschließend werden die Ergebnisse in den Schlussfolgerungen auch mit Hinblick auf den weiteren Forschungsbedarf dargestellt.

\section{Rahmenbedingungen der Einführung des Mindestlohns}

Im Unterschied zu anderen Branchen mit einem Mindestlohn zeichnet sich die Pflegebranche durch einige Merkmale aus, die eine herkömmliche Anwendung des AEntG über die Allgemeinverbindlicherklärung eines branchenweiten Tarifvertrags nicht zulassen. Die Pflegebranche ist durch einen sehr hohen Anteil kirchlicher Träger gekennzeichnet, die nicht dem traditionellen Tarifrecht unterliegen, sondern nach dem sogenannten Dritten Weg Löhne und Arbeitsbedingungen in paritätisch besetzten Arbeitsrechtlichen
Kommissionen aushandeln. ${ }^{1}$ Durch diese heterogene Trägerlandschaft ist es unmöglich, einen nach $\S 7$ AEntG repräsentativen Tarifvertrag zu finden, der für allgemeinverbindlich erklärt werden kann. Um dennoch Mindestlöhne in der Pflegebranche einführen zu können, wurde bei der Erneuerung des AEntG 2009 ein eigener Abschnitt „Arbeitsbedingungen in der Pflegebranche" (§§ 10-13 AEntG) eingefügt. Dieser Abschnitt regelt die Festlegung eines Mindestlohns durch eine achtköpfige Kommission, ${ }^{2}$ die aus jeweils zwei Vertreterinnen und Vertretern der vier in der Pflegebranche relevanten Interessensgruppen ( $\$ 12 \mathrm{AEntG}$ ) besteht. Diese Interessensgruppen sind die nichtkirchlichen Arbeitgeber, die Arbeitnehmerinnen und Arbeitnehmer sowie die kirchlichen Trägerverbände (Dienstgeber) und die entsprechenden Arbeitnehmervertretungen (Dienstnehmer). Der von der Pflegekommission vorgeschlagene Mindestlohn kann vom zuständigen Bundesministerium für Arbeit und Soziales (BMAS 2010) entweder umgesetzt oder abgelehnt werden. Eine politische Einflussnahme auf die Lohnhöhe ist folglich nicht möglich. Mit der Pflegearbeitsbedingungenverordnung (PflegeArbbV) vom 1. August 2010 wurde ein Mindestlohn in der Höhe von 8,50 Euro in Westdeutschland und Berlin ${ }^{3}$ sowie von 7,50 Euro in Ostdeutschland eingeführt.

Der betriebliche Geltungsbereich des Mindestlohns umfasst sämtliche Einrichtungen und Pflegedienste, die überwiegend Pflegeleistungen an Pflegebedürftige im Rahmen des elften Sozialgesetzbuches (SGB XI) anbieten. Explizit ausgeschlossen von der Regelung sind Krankenhäuser sowie Einrichtungen, die überwiegend ambulante Krankenpflegeleistungen erbringen. Weiterhin sind Einrichtungen ausgeschlossen, deren Hauptzwecke die medizinische Vorsorge, die medizinische Rehabilitation, Leistungen zur Teilhabe am Arbeits- beziehungsweise Gemeinschaftsleben sowie die Erziehung und schulische Ausbildung kranker oder behinderter Menschen sind ( $\$ 1$ Abs. 2 PflegeArbbV). Zudem ist die Pflege in privaten Haushalten nicht erfasst, wenn die Pflegekräfte von Privatpersonen angestellt sind und nicht als Beschäftigte eines Pflegedienstes arbeiten. Inwieweit der Mindestlohn Auswirkungen auf diese Bereiche hat, wird im Rahmen dieses Artikels nicht behandelt. Durch den persönlichen Geltungsbereich werden nach $\S 1$ Abs. 3 PflegeArbbV ausschließlich Pflegefach- und Pflegehilfskräfte erfasst. Dies impliziert, dass Beschäftigte, die hauptsächlich hauswirtschaftliche Tätigkeiten ausüben,

\footnotetext{
${ }^{1}$ Für eine ausführlichere Analyse des kirchlichen Arbeitsrechts siehe unter anderem Lührs (2010).

${ }^{2}$ Diese wird im Folgenden als Pflegekommission bezeichnet.

${ }^{3}$ In der Pflege gilt der westdeutsche Mindestlohn ebenfalls für Berlin. Im Folgenden wird bei den Beschreibungen Berlin zu Westdeutschland gezählt.
} 
nicht vom Mindestlohn erfasst werden. Zu diesen hauswirtschaftlichen Tätigkeiten gehören Einkaufen, Kochen, Putzen, Spülen oder Wäschewaschen. Weiterhin sind Auszubildende, Praktikantinnen und Praktikanten sowie Zivildienstleistende oder Freiwillige von den Regelungen ausgenommen.

\section{Methoden}

Bei der Evaluation von arbeitsmarktpolitischen Maßnahmen ist die Triangulation unterschiedlicher Methoden eine gängige Vorgehensweise. Bei dieser Untersuchung wurden quantitative und qualitative Methoden kombiniert. Neben amtlichen Daten und einer standardisierten Befragung wurden die Ergebnisse aus neun Fallstudien sowie 20 Experteninterviews ausgewertet. Durch die verwendete Methodenkombination konnten quantitative Ergebnisse durch Erkenntnisse aus qualitativen Interviews abgesichert und besser interpretiert werden. Zudem bestand die Möglichkeit, Hypothesen zu generieren und zeitnah in Interviews von Experten bewerten zu lassen.

\subsection{Qualitative Methoden}

Die meisten Interviews und Fallstudien wurden im Zeitraum April bis Juli 2011 geführt. Da der Mindestlohn in der Pflegebranche erst zum 1. August 2010 eingeführt wurde und somit relativ nah am Untersuchungszeitraum lag, waren die Untersuchungsbedingungen für die Experteninterviews und Fallstudien besonders günstig. Erinnerungsfehler sind dadurch weniger wahrscheinlich.

Innerhalb der qualitativen Untersuchungen wurden zwei methodische Ansätze kombiniert. Zunächst wurde nach dem Fallstudienkonzept von Apel (2009) vorgegangen. Dabei werden die Gespräche aufgezeichnet, transkribiert und anschließend zu Fallberichten verdichtet. Die Fallberichte sollen als Primärquelle für weitere Analysen dienen, wobei nicht mehr auf die Originalquellen zurückgegriffen werden muss. Im Laufe der Untersuchungen wurde jedoch zum einen der Mehrwert von Originalzitaten deutlich und zum anderen wurden die Leitfäden angepasst, indem die Leitfäden um Fragen, die sich als nicht relevant herausgestellt haben, reduziert beziehungsweise um Fragen erweitert wurden, die sich im Zuge der Interviews oder erster quantitativer Ergebnisse als bedeutend herausstellten. Es fand demnach kein rein chronologisches Vorgehen von Datenerhebung, Datenanalyse und Theoriebildung statt. Vielmehr wurde zwischen den unterschiedlichen Verfahren hin- und hergewechselt. Somit konnte den Bedürfnissen der Interviewpartner und -partnerinnen besser Rechnung getragen werden. Es wurde also das Fallstudienkonzept von Apel (2009) durch das Verfahren der Grounded Theory (Lamnek
2005) ergänzt. Eine Kombination dieser beiden Methoden ermöglicht eine genauere und umfassendere Darstellung der Sachverhalte, die in besonderem Maße auf die Sicht der Befragten eingeht.

Bei den Fallstudien wurden in verschiedenen Pflegeeinrichtungen Gespräche mit jeweils einem Vertreter oder einer Vertreterin des Arbeit- oder Dienstgebers, des Betriebsrats ${ }^{4}$ sowie Gruppen- beziehungsweise Einzelgespräche mit drei bis fünf Arbeitnehmern und Arbeitnehmerinnen beziehungsweise Dienstnehmern und Dienstnehmerinnen geführt. Bei stationären Pflegediensten wurde zudem der Heimbeirat befragt.

Die Einrichtungen, in denen Fallstudien durchgeführt wurden, wurden nach Trägerschaft, Art der Pflege sowie nach regionalen Kriterien ausgewählt. ${ }^{5}$ Bei der regionalen Auswahl wurde neben einer Differenzierung nach Westund Ostdeutschland ebenfalls nach der Arbeitsmarktsituation selektiert. Hierfür wurden die SGB III-Regionaltypen der Bundesagentur für Arbeit (BA 2010) verwendet. In Ostdeutschland wurde ebenfalls ein besonderes Gewicht auf Grenzregionen gelegt, um mögliche Einflüsse durch Pflegedienste aus den Nachbarländern zu berücksichtigen.

Die Fallstudien wurden aufgrund der Besonderheiten auf dem Markt für Pflegeleistungen um Expertengespräche ergänzt. Im Rahmen der Experteninterviews wurden mit Vertretern und Vertreterinnen von Arbeitgeberverbänden, Gewerkschaften, Dienstgeberverbänden und von Dienstnehmerverbänden Gespräche geführt. Dabei wurden die betreffenden Personen nicht ausschließlich in ihrer Funktion als Vertreter oder Vertreterinnen der entsprechenden Interessensgruppen interviewt, sondern zugleich in ihrer Funktion als Mitglieder der Pflegekommission. Weiterhin wurden mit zwei Vertretern und Vertreterinnen der Pflegekassen Gespräche geführt, da diese die Pflegesätze aushandeln und somit Einfluss auf die Preisgestaltung im Pflegebereich besitzen. Zu Themen der Qualitätssicherung wurden Experten und Expertinnen des Medizinischen Dienstes der Krankenkassen auf Bundesebene sowie auf Landesebene in einem ostdeutschen und einem westdeutschen Bundesland befragt. Um die Sichtweise der Pflegebedürftigen zu berücksichtigen, wurden Gespräche mit Vertretern und Vertreterinnen von Interessenverbänden der zu Pflegenden geführt. Ebenfalls fanden vier Gespräche mit den Geschäftsführungen neugegründeter Einrichtungen statt. Ziel war es, zu erfahren, ob bei Einrichtungen, deren Gründungsidee kurz vor oder nach Bekanntgabe der Mindestlohneinführung entstand, die

\footnotetext{
${ }^{4}$ Betriebsrat und Heimbeirat konnten nur interviewt werden, wenn die untersuchte Einrichtung einen Betriebsrat beziehungsweise Heimbeirat hatte.

${ }^{5}$ Aufgrund der geringen Bereitschaft vieler Einrichtungen, sich an einer Fallstudie zu beteiligen, musste das gewählte Untersuchungskonzept leicht angepasst werden und statt der anvisierten neun Regionen wurden lediglich in sieben Regionen Fallstudien durchgeführt.
} 
Einführung des Mindestlohns Auswirkungen auf die Gründung hatte. Zur Berücksichtigung der Veränderungen bei der Entsendung ausländischer Arbeitnehmer und Arbeitnehmerinnen - insbesondere nach der Einführung der vollständigen Arbeitnehmerfreizügigkeit für acht Mitgliedsstaaten der Europäischen Union (EU) am 1. Mai 2011 - fand ein Interview mit der für deren Erfassung zuständigen Zentrale Auslands- und Fachvermittlung statt. Schließlich wurden noch vier Gespräche mit Vertretern und Vertreterinnen der Finanzkontrolle Schwarzarbeit sowohl in Hauptzollämtern als auch in der Koordinierungsstelle in Köln geführt, um Einblicke in die Kontrollpraxis sowie über die Häufigkeit und Formen von Verstößen insbesondere in Grenzregionen zu osteuropäischen EU-Nachbarländern zu gewinnen.

Auf Basis eines vorab erarbeiteten Fragen- und Themengerüsts wurden differenzierte Leitfäden für die verschiedenen Adressatengruppen entwickelt. Es wurde während der Interviews darauf geachtet, flexibel auf die jeweiligen Akteure einzugehen, so dass auch wichtige Aspekte, die über die Leitfäden hinausgingen, miteinbezogen werden konnten.

Dabei wurden die Fallstudien (mit einer Ausnahme) ${ }^{6}$ vor Ort durchgeführt. Die Experten und Expertinnen wurden ausschließlich telefonisch befragt. Die geführten Interviews wurden aufgezeichnet und anschließend transkribiert.

\subsection{Quantitative Methoden}

Die Entwicklung der Pflegebranche seit 2003 lässt auf eine geringe konjunkturelle Abhängigkeit schließen. Wie später detaillierter diskutiert wird, ist seit 2003 ein kontinuierliches Wachstum der Branche zu beobachten. Gleichzeitig haben mehrere Studien (Afentakis und Maier 2010; RWI et al. 2009) einen sehr starken Zusammenhang der Entwicklung der Pflegebranche mit dem demographischen Wandel hergestellt. Dieser Zusammenhang ist in anderen Branchen nicht oder lediglich in einem geringeren Ausmaß gegeben. Diese starke Abhängigkeit von der demographischen Entwicklung schließt einen Großteil der Branchen als mögliche Kontrollbranchen aus.

Eine naheliegende Kontrollbranche aus dem Gesundheitssektor wären Krankenhäuser. Diese beschäftigen Krankenpfleger und -pflegerinnen sowie Krankenpflegehelfer und -pflegehelferinnen, deren Tätigkeitsbereich dem von Pflegekräften sehr ähnlich ist. Zudem kann bei Krankenhäusern ebenfalls ein ähnlicher Zusammenhang der Branchenentwicklung mit dem demographischen Wandel vermutet werden. Ein Vergleich der Entwicklung von Krankenhäusern und Pflegeeinrichtungen in IAW (2011) zeigt allerdings, dass die Common-Trends-Annahme nicht aufrecht erhalten werden kann, da der Krankenhaussektor in

\footnotetext{
${ }^{6}$ Aus betriebsinternen Gründen musste eine Fallstudie in Form mehrerer Telefonate durchgeführt werden.
}

den letzten Jahren deutlich geschrumpft ist. Daher kommen auch Krankenhäuser, aufgrund der sehr unterschiedlichen Entwicklung der beiden Branchen, als potenzielle Kontrollbranche nicht in Frage.

Seit der Studie durch Card und Krueger (1994) haben sich binäre Differenz-von-Differenz-Verfahren (DvD) in der mikroökonometrischen Wirkungsanalyse von Mindestlöhnen als Standard etabliert. Dies setzt allerdings voraus, dass eine geeignete Kontrollgruppe identifiziert werden kann. Wie bereits erwähnt ist ein Vergleich der Pflegebranche mit einer vergleichbaren Branche ohne Mindestlohn aufgrund der eingangs erwähnten Besonderheiten schwierig. Alternativ kann eine Kontrollgruppe innerhalb der Branche gesucht werden. Abhängig von den verfügbaren Daten können auch Beschäftigte mit Stundenlöhnen etwas oberhalb des Mindestlohns als Kontrollbeobachtungen dienen oder die Einrichtungen werden in Abhängigkeit von der Tarifbindung in Treatment- und Kontrollgruppe aufgeteilt.

Machin et al. (2003) haben in ihrer Untersuchung der Auswirkungen des Mindestlohns auf Pflegeheime in Großbritannien eine Alternative zum eben beschriebenen DvDAnsatz mit einer eindeutigen Zuordnung in Treatment- und Kontrollgruppe gewählt. Die Autoren verwenden statt einer binären Treatmentvariable ein kontinuierliches Maß. Die Grundidee ist, dass Änderungen in der abhängigen Variable auf die Unterschiede in der Treatmentvariable zurückzuführen sind. Sie nutzen dafür eine wiederholte Befragung von Pflegeheimen, um die Folgen der Mindestlohneinführung zu identifizieren.

$\Delta \ln Y_{i t}=\beta_{0}+\beta_{1} \mathrm{MIN}_{i, t-1}+\beta_{2} X_{i, t-1}+\eta_{i t}$

In Schätzgleichung (1) wird die Veränderung der logarithmierten Ergebnisvariable $(Y)$ zwischen einem Zeitpunkt vor Einführung des Mindestlohns $(t-1)$ und einem Zeitpunkt nach der Mindestlohneinführung $(t)$ anhand eines Maßes für die Betroffenheit des Mindestlohns ( $\left.\mathrm{MIN}_{i, t-1}\right)$ und weiteren erklärenden Variablen aus der Phase vor Mindestlohneinführung $\left(X_{i, t-1}\right)$, wie die Trägerschaft der Einrichtung, Tarifbindung und weiterer betrieblicher Indikatoren, erklärt.

Als Maß für die Betroffenheit können sowohl diskrete als auch kontinuierliche Maße verwendet werden. Als kontinuierliche Maße bieten sich ex ante Maße der Eingriffsintensität wie der Anteil der Beschäftigten einer Einrichtung, die weniger als den Mindestlohn bekommen, oder die Lohnlücke an. Als Lohnlücke wird hier der Anteil der Lohnsumme der Beschäftigten mit einer Entlohnung unterhalb des Mindestlohns an der gesamten Lohnsumme definiert:

$\operatorname{MIN}_{i, t-1}=\frac{\sum_{j} h_{j i} \max \left[W^{\min }-W_{j, 0}\right]}{\sum_{j} h_{j i} W_{j}}$

Im Zähler wird für sämtliche Lohngruppen unterhalb des Mindestlohnniveaus die Differenz zwischen der Mindestlohnhöhe $W^{\text {min }}$ und der Lohnhöhe $W_{j}$ der Lohngruppe $j$ 
mit der Anzahl der Beschäftigten der Einrichtung $i$ in dieser Lohngruppe $\left(h_{j i}\right)$ multipliziert und aufsummiert. Anschließend wird dieses Ergebnis durch die Lohnsumme aller Beschäftigten einer Einrichtung dividiert. Dieses Maß wird als Lohnlücke bezeichnet, da es misst, um wie viel die Lohnsumme steigen muss, damit ceteris paribus alle Beschäftigten mindestens den Mindestlohn erhalten.

Der Ansatz von Machin et al. (2003) kann ausschließlich mit Hilfe eines Paneldatensatzes durchgeführt werden, da die Ergebnisvariablen die Veränderung über den Zeitraum der Mindestlohneinführung messen. Voraussetzung für die Validität dieses Schätzansatzes ist die Gültigkeit der Exogenitätsannahme, welche vergleichbar mit der CommonTrends-Annahme beim Differenzen-von-Differenzen-Ansatz ist. Demnach darf die gewählte Treatmentvariable die Ergebnisvariable ausschließlich durch die Mindestlohneinführung beeinflussen. Dies schließt unterschiedliche Zeittrends zwischen den Einrichtungen aus. Diese Annahme ist relativ stark und verlangt, dass die Veränderung des Lohnniveaus und der Beschäftigung von der Lohnstruktur zum vorherigen Messzeitpunkt unabhängig ist. Das heißt, dass eine relativ niedrige Entlohnung im Ausgangszeitpunkt keinen Einfluss auf die künftige Entwicklung der Löhne oder der Beschäftigung hat. Diese Annahme könnte beispielsweise dann verletzt sein, wenn ein „Regression-to-the-mean“Phänomen vorliegt. Hier würde die Treatmentvariable nicht nur den Einfluss des Mindestlohns messen, sondern ebenfalls den vom Mindestlohn unabhängigen Trend, dass Einrichtungen mit niedrigeren Stundenlöhnen höhere Lohnsteigerungen aufweisen als Einrichtungen mit höheren Löhnen.

Die Gültigkeit dieser Annahme muss überprüft werden, um die Belastbarkeit der Ergebnisse einschätzen zu können. Hier schlagen Machin et al. (2003) ein Placebo-Experiment vor, in dem derselbe Schätzansatz für einen Zeitraum ohne eine Mindestlohneinführung durchgeführt wird. Ein Vergleich der Koeffizienten ermöglicht es, mögliche Effekte des Mindestlohns zu identifizieren.

\section{Beschreibung des Paneldatensatzes}

Durch die erst kürzlich stattgefundene Einführung des Mindestlohns in der Pflegebranche liegen noch keine amtlichen Daten für den Zeitraum nach der Mindestlohneinführung vor. Daher können auf Basis amtlicher Daten noch keine Wirkungsanalysen durchgeführt werden. Folglich wird für die Wirkungsanalysen in dieser Studie eine von T.I.P. Biehl $\&$ Partner im Auftrag des BMAS durchgeführte wiederholte assistierte Onlinebefragung von Pflegeeinrichtungen verwendet. $^{7}$

\footnotetext{
${ }^{7}$ Im Folgenden wird diese Befragung als T.I.P.-Pflegebefragung bezeichnet.
}

Im Rahmen dieser Befragung wurde aus dem Adressmaterial der Pflegestatistik eine nach Ost- und Westdeutschland, Art der Pflege sowie Trägerart geschichtete Zufallsstichprobe gezogen. Dies sollte gewährleisten, dass für differenzierte Auswertungen anhand dieser Merkmale ausreichend Fallzahlen zur Verfügung stehen. Die Erhebung der ersten Welle fand im Herbst 2010, die der zweiten im Frühjahr 2011 statt. $^{8}$ Dabei bezog sich die erste Welle retrospektiv auf den Zeitraum vor der Einführung des Mindestlohns.

Insgesamt haben sich 1.355 Einrichtungen an der ersten und 731 Einrichtungen an der zweiten Welle der Befragung beteiligt. Allerdings haben nicht alle Einrichtungen den Fragebogen beendet. Da für die Evaluation der Mindestlohnwirkungen die Lohnstruktur das zentrale Merkmal ist, wurden aus den Analysen sämtliche Einrichtungen ausgeschlossen, welche die Fragen zur Lohnstruktur nicht beantwortet haben.

Die Lohnstruktur liegt für insgesamt 1.110 Einrichtungen in mindestens einer der beiden Befragungswellen vor. Dies entspricht einer Rücklaufquote von 6,4\%. Weitere Plausibilitätschecks ergaben, dass für drei Einrichtungen inkonsistente Angaben vorliegen, die daher aus der weiteren Betrachtung ausgeschlossen wurden. Von den verbliebenen 1.107 Einrichtungen haben 499 ausschließlich an der ersten, 207 ausschließlich an der zweiten und 401 Einrichtungen an beiden Wellen teilgenommen. Während also für Analysen mit der ersten beziehungsweise zweiten Welle 900 beziehungsweise 608 Einrichtungen zur Verfügung stehen, können lediglich 401 Einrichtungen für Analysen mit dem Paneldatensatz verwendet werden.

Der Kern der Befragung war eine detaillierte Abfrage der Stundenlöhne der Pflegekräfte. Die Stundenlöhne wurden zwischen 6 und 14 Euro erfasst. Dabei wurden Lohngruppen von jeweils 0,50 Euro gebildet. Die Einrichtungen sollten für Pflegehilfs- und Pflegefachkräfte getrennt und differenziert nach Geschlecht die Anzahl der Beschäftigten in den einzelnen Lohngruppen angeben. Neben der Abfrage der Lohnstruktur enthielt der Fragebogen noch Fragen zu allgemeinen Charakteristika der Einrichtungen, zur Beschäftigtenstruktur, zur Kostenstruktur, zum Ertrag und zu offenen Stellen sowie zur Wirkung des Mindestlohns.

Bei der Verwendung von Befragungsdaten, insbesondere bei geringen Rücklaufquoten, muss berücksichtigt werden, dass die Bereitschaft, an der Befragung teilzunehmen, selektiv sein kann. Diese Selektion kann anhand bekannter Merkmale der Untersuchungseinheiten analysiert werden. Für die vorliegende Befragung sind über die nicht teilnehmenden Einrichtungen lediglich die Schichtungsmerkmale

${ }^{8}$ Eine ausführliche Dokumentation der Befragung befindet sich im Zwischen- und Endbericht des Befragungsinstituts (T.I.P. Biehl \& Partner 2010 \& 2011). 
Region, Art der Pflege und Trägerart bekannt. Daher beschränken sich die folgenden vergleichenden Analysen zwischen teilnehmenden und nicht teilnehmenden Einrichtungen auf diese Merkmale. Diese Analysen zeigen, dass insbesondere westdeutsche und stationäre Einrichtungen eine höhere Rücklaufquote haben: 64,3 \% (63,2 \%) der Einrichtungen in der Nettostichprobe der ersten (zweiten) Welle sind aus Westdeutschland, während dieser Anteil in der Bruttostichprobe $58,6 \%$ beträgt. Noch stärker sind die Unterschiede im Antwortverhalten zwischen ambulanten und stationären Einrichtungen. Der Anteil der stationären Einrichtungen in der Bruttostichprobe ist mit 35,2 \% vergleichsweise niedrig. In der Nettostichprobe ist die Verteilung zwischen ambulanten und stationären Einrichtungen fast ausgeglichen. In der ersten (zweiten) Welle sind 48,9 \% (47,3\%) der Einrichtungen stationär.

Neben einem Vergleich zwischen den an der Befragung teilnehmenden und nicht teilnehmenden Einrichtungen ermöglicht die Konzeption der Befragung als Panelbefragung mit einer erweiterten zweiten Welle eine mögliche Selektion unter den an mindestens einer Welle teilnehmenden Einrichtungen anhand von Merkmalen aus der Befragung zu identifizieren.

Die Analysen zeigen, dass vor allem die Anzahl der Beschäftigten die Teilnahmebereitschaft an der zweiten Welle signifikant beeinflusst. Insbesondere in kleinen Einrichtungen mit maximal zehn Mitarbeiterinnen und Mitarbeitern ist die erneute Teilnahmebereitschaft signifikant höher. Dies ist ein Indiz, dass die detaillierte Abfrage der Lohnstruktur, die auch in der zweiten Welle stattfand, insbesondere größere Einrichtungen von einer erneuten Teilnahme abgeschreckt hat. Neben der Beschäftigtenanzahl haben insbesondere Einrichtungen, die eine schlechte Ertragslage haben, die vom Mindestlohn eine schnellere und leichtere Stellenbesetzung erwarten oder die planen, die Pflegesätze aufgrund der Mindestlohneinführung neu zu verhandeln, eine signifikant höhere erneute Teilnahmewahrscheinlichkeit. Im Gegensatz dazu haben Einrichtungen, die vom Mindestlohn eine Qualitätssteigerung der erbrachten Pflegeleistungen erwartet haben, eine signifikant niedrigere Teilnahmewahrscheinlichkeit in der zweiten Welle. Die Ergebnisse sind ein Indiz, dass insbesondere Einrichtungen, die von der Mindestlohneinführung stärker betroffen sind, ein Interesse am Gegenstand der Befragung hatten und daher an beiden Wellen teilgenommen haben.

Eine Analyse der Teilnahmewahrscheinlichkeit an der ersten Welle auf Basis der Informationen aus der zweiten Welle ergab, dass im Vergleich zu privaten Trägern Einrichtungen in freigemeinnütziger Trägerschaft mit einer höheren Wahrscheinlichkeit neben einer Teilnahme an der zweiten Welle auch an der ersten Welle teilgenommen haben. Im Zusammenhang mit der allgemein sehr viel geringeren Teilnahmebereitschaft der freigemeinnützigen Träger zeigt dies, dass die Entscheidung, nicht an der Befragung teilzunehmen vor der ersten Welle getroffen wurde. Weiterhin haben Einrichtungen mit einer unabhängigen Lohngestaltung sowie mit Planungen, Pflegehilfskräfte oder sonstiges Personal einzustellen, eine signifikant höhere Teilnahmewahrscheinlichkeit an der ersten Welle. Die Meinung in der zweiten Welle, dass sich das Aufgabenspektrum der Mitarbeiter und Mitarbeiterinnen durch den Mindestlohn erweitert hat, senkt die Wahrscheinlichkeit, dass die Einrichtung bereits an der ersten Welle teilgenommen hat. Eine mögliche Erklärung ist, dass einige Einrichtungen an der Befragung teilnehmen wollten, aber die Teilnahme an der ersten Welle aufgrund einer Neustrukturierung und Organisation der Einrichtung im Rahmen der Mindestlohneinführung verpasst haben. ${ }^{9}$

Die Selektivität im Paneldatensatz muss bei der Interpretation der Ergebnisse berücksichtigt werden. Insbesondere der Entscheidungsprozess, überhaupt an der Befragung teilzunehmen, kann nicht beobachtet werden, ist aber wahrscheinlich stark von der Betroffenheit der Einrichtungen vom Mindestlohn beeinflusst. Inwieweit eher die vom Mindestlohn stark betroffenen Einrichtungen oder eher die Einrichtungen antworten, die sich vom Mindestlohn einen Schutz vor Konkurrenz mit niedrigen Löhnen erhoffen, lässt sich anhand der Daten nicht abschließend klären. Ein Vergleich der Befragungsergebnisse mit der Pflegestatistik hinsichtlich der Beschäftigtenstruktur weist allerdings keine großen Unterschiede auf (IAW 2011, S. 74-81). Dies kann als ein Indiz gewertet werden, dass zumindest hinsichtlich der vergleichbaren Indikatoren keine großen Verzerrungen im Befragungsdatensatz vorliegen.

Die durchgeführten Selektivitätsanalysen geben Hinweise darauf, dass Einrichtungen, die vom Mindestlohn stärker betroffen waren, eine höhere erneute Teilnahmebereitschaft haben. Dies kann in den Schätzungen zu einer Überschätzung der Effekte führen, wenn vom Mindestlohn stark betroffene Einrichtungen eher an beiden Befragungswellen teilgenommen haben.

Für die Auswertungen wurde anhand der Schichtungsmerkmale ein Designgewicht generiert, das eine Hochrechnung auf die Grundgesamtheit ermöglicht. Zudem wurden die Ergebnisse der Selektivitätsanalyse für eine Neuberechnung der Gewichtungsfaktoren verwendet. Dabei wurden die Designgewichte mit den berechneten Teilnahmewahrscheinlichkeiten mit der jeweils anderen Welle multipliziert.

\section{Weitere Datenquellen}

Die Pflegebranche hat gegenüber anderen Branchen den Vorteil, dass mit der Pflegestatistik ein sehr umfangreicher

\footnotetext{
${ }^{9}$ Die erste Befragung fand kurz nach Einführung des Mindestlohns im September/Oktober 2010 statt.
} 
Datensatz der amtlichen Statistik für die Branche zur Verfügung steht. Die Pflegestatistik wird seit 1999 von den statistischen Ämtern des Bundes und der Länder im Zweijahresrhythmus erhoben. Sie ist eine Vollerhebung der Einrichtungen im Pflegebereich sowie sämtlicher Pflegebedürftiger. ${ }^{10}$ Für die Analysen im Rahmen dieser Studie werden ausschließlich die Informationen zu den Pflegeeinrichtungen der Jahre 2003 bis 2009 verwendet.

Die Pflegestatistik enthält sowohl umfangreiche Informationen über die Pflegeeinrichtungen als auch über die Beschäftigten in den jeweiligen Einrichtungen. Folglich lassen sich detaillierte Aussagen über das Angebot von Pflegeleistungen, über die Struktur der Branche sowie zu den Beschäftigten in der Branche machen.

Da die gesetzliche Definition von Pflegeeinrichtungen, die auch vom Statistisches Bundesamt (2007) zur Abgrenzung der Pflegestatistik verwendet wird, weiter gefasst ist als die Definition des betrieblichen Geltungsbereichs des Mindestlohns in der Pflege, beziehen sich die folgenden Auswertungen auf eine Subpopulation der Pflegestatistik. ${ }^{11}$ Für die Analysen wurden ausschließlich Einrichtungen berücksichtigt, die überwiegend ältere Menschen betreuen. Auch der persönliche Geltungsbereich lässt sich in der Pflegestatistik durch die überwiegende Tätigkeit jedes Beschäftigten abgrenzen. Trotz der sehr umfangreichen Datenbasis der Pflegestatistik ist sie für Wirkungsanalysen des Mindestlohns nicht verwendbar, da sie weder Lohn- noch Ertragsinformationen enthält. Zusätzlich ist es kaum möglich, innerhalb der Pflegestatistik geeignete Kontrollbeobachtungen zu finden, da $91 \%$ der Einrichtungen in der Pflegestatistik unter den betrieblichen Geltungsbereich fallen. Daher wird die Pflegestatistik ausschließlich für deskriptive Analysen verwendet.

Als zusätzliche Datenquellen für weitere Auswertungen wurde das Betriebs-Historik-Panel (BHP), die Integrierten Erwerbsbiographien (IEB) - beide Datensätze werden durch das Institut für Arbeitsmarkt- und Berufsforschung (IAB) zur Verfügung gestellt - sowie der Mikrozensus des Statistischen Bundesamtes verwendet. Auf Grundlage des BHP wurde aus den Wirtschaftszweigen „Altenpflegeheime (WZ03: 85.31.5)“, „Einrichtungen zur Eingliederung und Pflege Behinderter (WZ03: 85.31.7)“12 sowie „Ambulante

\footnotetext{
${ }^{10}$ Die Statistiken zu den Pflegeeinrichtungen und zu den Pflegebedürftigen werden separat erhoben und dürfen aus Datenschutzgründen nicht verknüpft werden.

${ }^{11}$ Die Pflegestatistik berücksichtigt ebenfalls Pflegeheime für behinderte oder psychisch kranke Menschen sowie Pflegedienste, die nicht überwiegend Leistungen nach SGB XI anbieten.

${ }^{12} \mathrm{Da}$ sich nachträglich herausstellte, dass der Wirtschaftszweig „Einrichtungen zur Eingliederung und Pflege Behinderter (WZ03: 85.31.7)“ nicht vom Mindestlohn betroffen ist, wurde dieser in den Auswertungen nicht berücksichtigt.
}

Soziale Dienste (WZ03: 85.32.6)“ eine nach Ost- und Westdeutschland sowie nach den Wirtschaftszweigen geschichtete Stichprobe von 4.000 Einrichtungen gezogen. Ziehungszeitpunkt war das Jahr 2008. Zu diesen Einrichtungen wurden sämtliche Individualdaten aus den IEB zur Verfügung gestellt.

\section{Deskriptive Analysen}

Die Pflegebranche ist durch zwei sehr unterschiedliche Bereiche, die stationäre sowie die ambulante Pflege, gekennzeichnet. Im Folgenden werden stationäre Einrichtungen als Pflegeheime und ambulante Einrichtungen als Pflegedienste bezeichnet. Dabei setzt sich die Branche etwa zu gleichen Anteilen aus Pflegeheimen (10.818) und Pflegediensten (10.752) zusammen. ${ }^{13}$ Allerdings unterscheiden sich die beiden Pflegebereiche hinsichtlich der Trägerschaften, der Betriebsgrößen sowie der Betreuungsverhältnisse. Berechnungen auf Basis der Pflegestatistik 2009 ergeben, dass $61,9 \%$ der Pflegedienste und 39,9\% der Pflegeheime in privater Trägerschaft sind. Dabei sind private Einrichtungen deutlich kleiner als Einrichtungen anderer Trägerarten. Lediglich 49,8\% der Beschäftigten bei Pflegediensten und $31,8 \%$ der Beschäftigten in Pflegeheimen arbeiten bei privaten Pflegeanbietern. Im Vergleich dazu sind lediglich 19,4\% der Pflegedienste und 30,2 \% der Pflegeheime in kirchlicher Trägerschaft, die aber $27,4 \%$ der Pflegekräfte bei Pflegediensten sowie $36,7 \%$ bei Pflegeheimen beschäftigen.

Seit 2003 hat sich die Anzahl der Einrichtungen um jährlich etwa 2,4 \% erhöht. Gleichzeitig ist die Beschäftigung von Pflegekräften in Vollzeitäquivalenten um jährlich 2,8 \% gestiegen, ${ }^{14}$ während die Anzahl der Pflegebedürftigen um jährlich 3,0 \% gewachsen ist. Die Branche wächst demnach nicht nur hinsichtlich der Anbieter von Pflegeleistungen. Auch die durchschnittliche Beschäftigtenzahl pro Einrichtung sowie die Anzahl der betreuten Pflegebedürftigen pro Einrichtung ist gestiegen. Dies entspricht der Wahrnehmung der Befragten in den Expertengesprächen und Fallstudien, in denen von einer Konzentration der Branche hin zu größeren Einrichtungen die Rede war. Die Konzentration wird einerseits als Folge der Mindestlohneinführung, andererseits auch als allgemeiner und vom Mindestlohn unabhängiger Trend in der Pflegebranche wahrgenommen. In beiden Fällen wird ein Finanzierungsproblem kleinerer Einrichtungen prognostiziert, das Ausgangspunkt für die stärkere Konzentration der Branche ist.

\footnotetext{
${ }^{13}$ Berechnungsgrundlage sind sämtliche Einrichtungen in der Pflegestatistik 2009, die überwiegend Leistungen nach SGB XI anbieten.

${ }^{14}$ Als Berechnungsgrundlage der Vollzeitäquivalente dienten die Anzahl der Pflegekräfte in Vollzeit, Teilzeit und geringfügiger Beschäftigung aus der Pflegestatistik 2003-2009 sowie berechnete Arbeitszeiten für diese Gruppen aus dem Mikrozensus.
} 
Abb. 1 Entwicklung der nominalen Bruttotagesentgelte in der Pflegebranche 2002-2008. Anmerkung: Die Berechnung geschieht ausschließlich auf Basis der Vollzeitbeschäftigten. Quelle: Eigene Berechnungen auf Basis des BHP 2002-2008

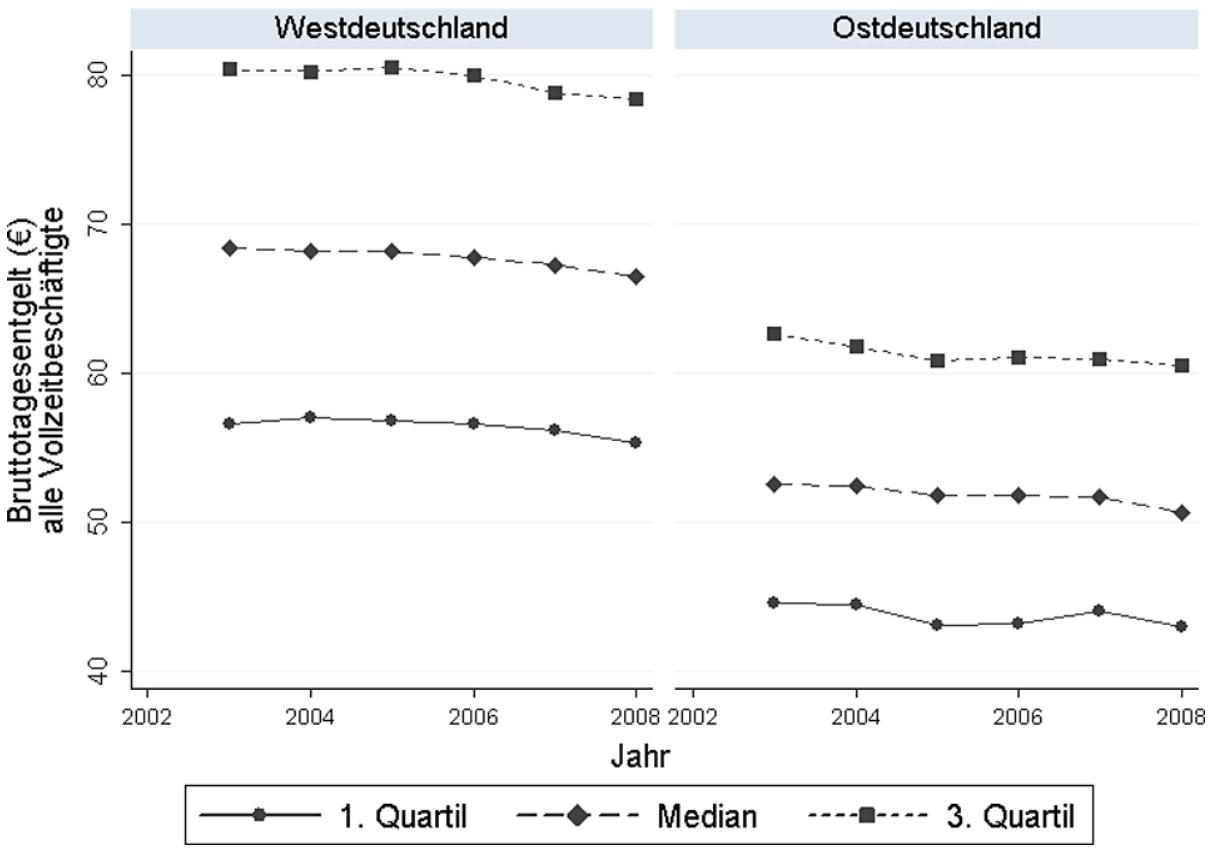

Das kontinuierliche Wachstum der Einrichtungen stellt diese vor das Problem, ausreichend qualifiziertes Personal für ihre steigende Nachfrage nach Pflegekräften zu finden. In der T.I.P.-Pflegebefragung berichten 84,3\% der Einrichtungen über Schwierigkeiten, geeignete Pflegefachkräfte zu finden. Dabei ist der Hauptgrund, dass zu wenige oder gar keine geeigneten Bewerbungen eingehen. ${ }^{15}$ Dies führt dazu, dass es 2011 pro Einrichtung durchschnittlich 2,2 offene Stellen gab. Dabei planten die Einrichtungen, in den nächsten zwölf Monaten im Schnitt 2,4 Pflegefachkräfte neu einzustellen. Auch in den qualitativen Untersuchungen wurde durchgängig, sowohl in den Expertengesprächen als auch in den Fallstudien, der Mangel an Pflegefachkräften genannt. Zudem wurde in einem Teil der Einrichtungen eine Unterbesetzung thematisiert. Der Fachkräftemangel habe sich insbesondere in den letzten Jahren verschlimmert. Dies sei vor fünf Jahren noch kein Problem gewesen. Dennoch haben Afentakis und Maier (2010) schon für 2005 einen Fachkräftemangel festgestellt, der allerdings noch vergleichsweise einfach durch Umschulung von Fachkräften aus dem Gesundheitsbereich gedeckt werden konnte.

Wenn man die demographische Entwicklung in den nächsten Jahren berücksichtigt, wird sich diese Situation weiter verschärfen. Bis 2030 ist zu erwarten, dass die Altersgruppe der über 65-jährigen um ein Drittel steigen wird, während die Altersgruppen der erwerbsfähigen Personen (zwischen 20 und 65 Jahren) um $15 \%$ und der Kinder und Jugendlichen (unter 20 Jahre) um $17 \%$ schrumpfen werden (Statistische Ämter des Bundes und der Länder 2011).

\footnotetext{
${ }^{15}$ Die Ergebnisse wurden im Rahmen der T.I.P.-Pflegebefragung ermittelt. Für ausführlichere Gründe siehe IAW (2011).
}

Die daraus resultierende steigende Nachfrage nach Pflegeleistungen wird sich nach Prognosen von RWI et al. (2009) weiter verstärken.

Aufgrund der beschriebenen Situation stellt sich der Arbeitsmarkt als Anbietermarkt für Pflegekräfte dar. Diese Entwicklung wurde auch in den qualitativen Untersuchungen bestätigt. Es wurde berichtet, dass Pflegefachkräfte bei der Stellensuche zwischen verschiedenen Arbeitgebern wählen können und zunehmend beim Stellenwechsel auf eine höhere Entlohnung achten. Dennoch scheint dies in der Pflegebranche nur bedingt zu einem Anstieg der Löhne zu führen. Auswertungen mit Hilfe des BHP in Abb. 1 zeigen, dass die nominalen Bruttotagesentgelte zwischen 2002 und 2008 sowohl in West- als auch in Ostdeutschland sogar leicht gesunken sind. Um den Einfluss der möglicherweise veränderten Stundenzahl aus der Darstellung fernzuhalten, wurden nur die Vollzeitbeschäftigten berücksichtigt. Dabei ist zu beachten, dass die Pflegebranche durch einen hohen Anteil an Teilzeitarbeit gekennzeichnet ist. 2009 waren laut Pflegestatistik 36,4 \% der Beschäftigten Vollzeit tätig. Insbesondere bei Pflegediensten ist der Anteil der Vollzeitbeschäftigten sehr niedrig (29,0\%), da ein Großteil der Beschäftigten in Pflegediensten entweder Teilzeit $(55,0 \%)$ oder geringfügig $(16,0 \%)$ beschäftigt ist. Die Entwicklung der Bruttotagesentgelte zeigt, dass sich bisher weder das kontinuierliche Wachstum der Branche noch der zunehmende Fachkräftemangel in Lohnsteigerungen niedergeschlagen hat.

Ein möglicher Grund ist in der Situation auf dem Markt für Pflegeleistungen zu finden. Die Preise für Pflegeleistungen werden in der Regel nicht zwischen den Einrichtungen und den Pflegebedürftigen vereinbart, sondern die 

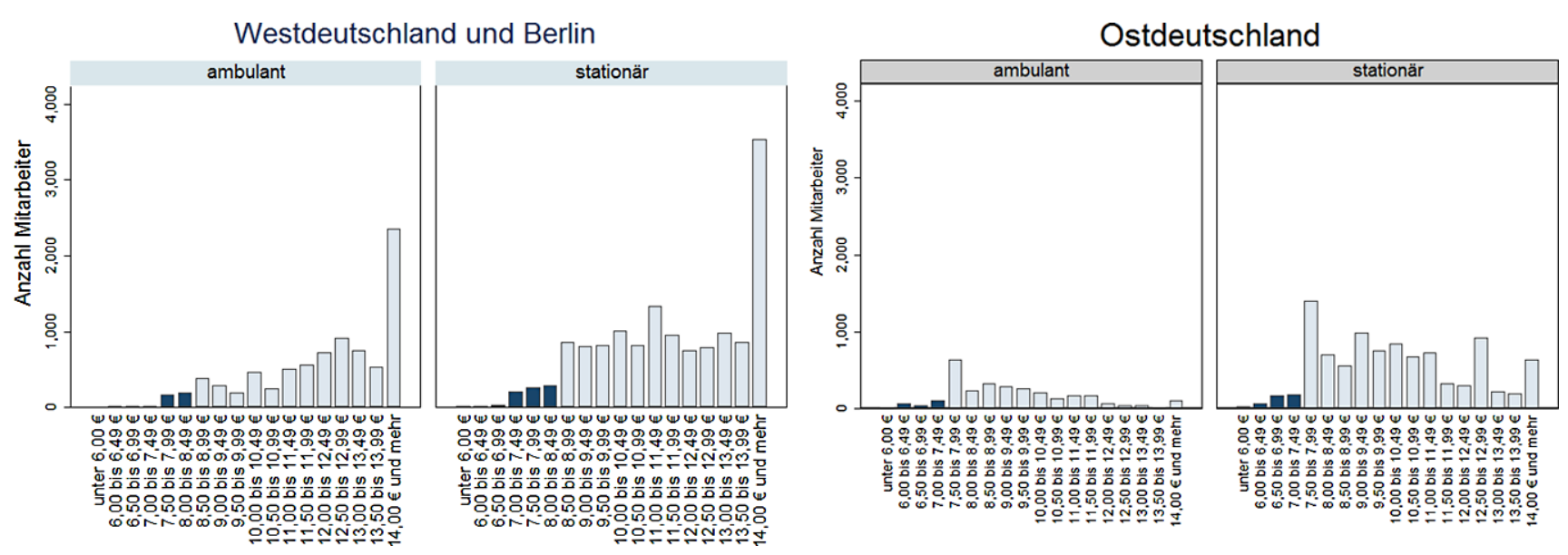

Abb. 2 Lohnverteilung vor Mindestlohneinführung. Quelle: Eigene Berechnungen auf Grundlage der T.I.P.-Pflegebefragung. Grundlage sind 578 (322) Einrichtungen und 24.182 (13.550) Beschäftigte in Westdeutschland (Ostdeutschland)

Einrichtungen verhandeln mit den Pflegekassen und Sozialversicherungsträgern. Dabei ist den Einrichtungen eine Preisdifferenzierung zwischen den unterschiedlichen Pflegekassen und Sozialversicherungsträgern gesetzlich nicht gestattet. Dementsprechend weist der Markt für Pflegeleistungen eine monopsonähnliche Situation auf, da ein großer einheitlicher Nachfrager - die Pflegekassen und Sozialversicherungsträger - vielen Anbietern gegenüber steht.

Eine Analyse der Entwicklung der Pflegesätze in Pflegeheimen zeigt, dass die Pflegesätze seit 2003 jährlich lediglich um 1,1-1,3\% in Westdeutschland und um 0,6$0,8 \%$ in Ostdeutschland gestiegen sind. ${ }^{16}$ Bei einer durchschnittlichen jährlichen Inflationsrate von 1,9\% im gleichen Zeitraum ist die Realvergütung für Pflegeleistungen sogar gesunken. ${ }^{17}$ Dies hat zur Konsequenz, dass die Einrichtungen Schwierigkeiten haben, höhere Löhne zu finanzieren, da es in einer personalintensiven Branche wie der Pflege neben den Personalkosten kaum Möglichkeiten gibt, Kosten einzusparen. Dementsprechend möchte laut T.I.P.Pflegebefragung ein Drittel der Einrichtungen in Westdeutschland sowie über die Hälfte der ostdeutschen Einrichtungen die Einführung des Mindestlohns nutzen, um höhere Pflegesätze auszuhandeln. Nach Ansicht der Medizinischen Dienste sowie der Pflegekassen ermöglichen die derzeit gültigen Pflegesätze jedoch eine Entlohnung nach beziehungsweise oberhalb des Mindestlohns, so dass aufgrund der Mindestlohneinführung die Beiträge künftig nicht angehoben werden.

\footnotetext{
${ }^{16}$ Auswertungen der Pflegestatistik 2003-2009.

${ }^{17}$ Quelle: Berechnungen auf Grundlage des Verbraucherpreisindex des Statistischen Bundesamtes. (http://www.destatis.de/jetspeed/portal/ $\mathrm{cms} /$ Sites/destatis/Internet/DE/Content/Statistiken/Zeitreihen/ WirtschaftAktuell/Basisdaten/Content100/vpi101a,templateId= renderPrint.psml [abgerufen am 27.08.2011]).
}

\section{Eingriffsintensität des Mindestlohns}

Die Wirkungen eines Mindestlohns werden entscheidend davon beeinflusst, wie stark der Mindestlohn in die Lohnstruktur eingreift. Da sich, wie bereits beschrieben, die Situation in Pflegediensten und -heimen unterscheidet, bietet es sich an, die Lohnverteilungen separat für die beiden Bereiche darzustellen.

Abbildung 2 zeigt anhand der T.I.P.-Pflegebefragung, wie sich die Lohnverteilung in West- und Ostdeutschland vor Einführung des Mindestlohns in den beiden Pflegebereichen darstellt. Die Lohnverteilungen in Westdeutschland zeigen deutlich, dass die Mehrheit der Beschäftigten in Westdeutschland einen Stundenlohn von 14 Euro oder mehr bekommt. Dennoch liegen die niedrigsten gezahlten Stundenlöhne teilweise deutlich unterhalb des Mindestlohnniveaus von 8,50 Euro. Insbesondere bei Pflegeheimen ist ein deutlicher Sprung in der Lohnverteilung bei der Mindestlohnhöhe zu erkennen. Dies ist zum einen auf Antizipationseffekte zurückzuführen, das heißt einige Einrichtungen haben bereits vor Einführung des Mindestlohns ihre Lohnstrukturen angepasst. Zum anderen liegt die niedrigste tarifliche Lohngruppe eines freigemeinnützigen Trägerverbandes ungefähr auf der Höhe des eingeführten Mindestlohns. ${ }^{18}$ Allerdings ist nicht auszuschließen, dass dieser Sprung zumindest teilweise auf sozial erwünschtes Antwortverhalten zurückzuführen ist, da die Befragung kurz nach Einführung des Mindestlohns durchgeführt wurde.

Die Lohnverteilung in Ostdeutschland zeigt einen deutlich stärker ausgeprägten sprunghaften Anstieg der Lohnverteilung in der Lohngruppe direkt oberhalb des Mindestlohns

\footnotetext{
${ }^{18}$ Recherchiert wurden die Entlohnungen der Trägerverbände Arbeiterwohlfahrt, Deutscher Caritasverband, Deutscher Paritätischer Wohlfahrtsverband, Deutsches Rotes Kreuz und Diakonisches Werk.
} 

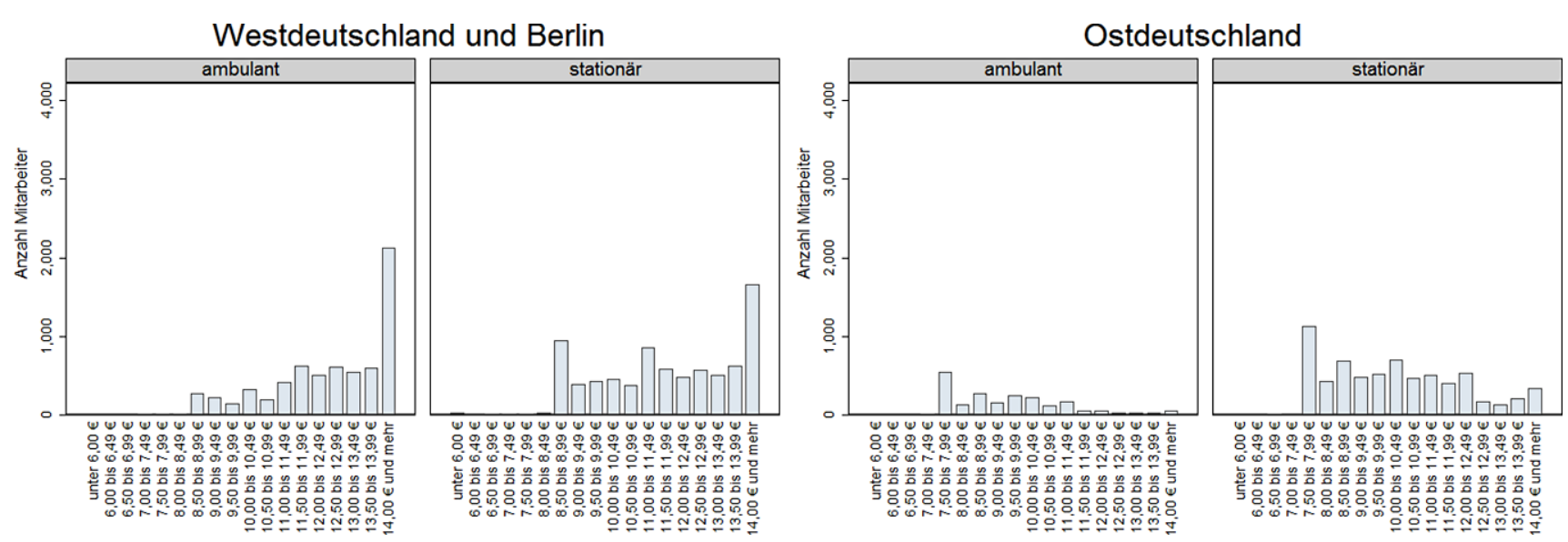

Abb. 3 Lohnverteilung nach Mindestlohneinführung. Quelle: Eigene Berechnungen auf Grundlage der T.I.P.-Pflegebefragung. Grundlage sind 383 (225) Einrichtungen und 15.893 (9.326) Beschäftigte in Westdeutschland (Ostdeutschland)

Tab. 1 Medianlöhne aufgeteilt nach Mindestlohntarifgebiet und Art der Pflege

Quelle: Eigene Berechnungen auf Grundlage der T.I.P.-Pflegebefragung

\begin{tabular}{llllll}
\hline & \multicolumn{2}{l}{ Vor Mindestlohneinführung } & & \multicolumn{2}{l}{ Nach Mindestlohneinführung } \\
\cline { 2 - 3 } & Pflegedienste & Pflegeheime & & Pflegedienste & Pflegeheime \\
\hline Westdeutschland und Berlin & 12,75 Euro & 12,25 Euro & & 13,25 Euro & 11,75 Euro \\
Ostdeutschland & 9,25 Euro & 10,25 Euro & & 9,75 Euro & 10,25 Euro \\
\hline
\end{tabular}

als in Westdeutschland. In beiden Pflegebereichen befindet sich der Modus der Verteilung in der Mindestlohngruppe. Im Unterschied zur Lohnverteilung in Westdeutschland spielt die Rechtszensierung der Lohnverteilung bei 14 Euro in Ostdeutschland eine untergeordnete Rolle. Die Lohnverteilungen zeigen, dass die Entlohnung in Ostdeutschland deutlich unterhalb der Entlohnung in Westdeutschland liegt.

Abbildung 3 zeigt die Lohnverteilung nach Einführung des Mindestlohns. Ähnlich wie bei den Lohnverteilungen vor Mindestlohneinführung ist in Ostdeutschland die Mindestlohngruppe der Modus der Verteilung. In Westdeutschland befindet sich der Modus beider Verteilungen erneut in der höchsten Lohngruppe. Allerdings ist die Mindestlohngruppe bei westdeutschen Pflegeheimen deutlich stärker besetzt als vor Mindestlohneinführung.

Durch die Abschneidegrenze bei 14 Euro ist eine Berechnung des arithmetischen Mittels, und vor allem eine Berechnung der Eingriffsmaße auf Basis des arithmetischen Mittels, nicht möglich. Stattdessen wurde der Medianlohn verwendet. Dieser ist, wie in Tab. $1 \mathrm{zu}$ sehen ist, in Ostdeutschland deutlich niedriger als in Westdeutschland. Der Unterschied ist bei Pflegediensten stärker ausgeprägt als bei Pflegeheimen, da der Medianlohn in westdeutschen Pflegediensten etwas höher ist als in Pflegeheimen, während der Medianlohn in ostdeutschen Pflegediensten ein Euro niedriger ist als in ostdeutschen Pflegeheimen. Nach der Mindestlohneinführung ist der Medianlohn der Pflegedienste angestiegen. In Pflegeheimen blieb dieser konstant beziehungs- weise ist leicht gesunken. Allerdings lässt sich nicht genau sagen, inwieweit der Medianlohn tatsächlich konstant geblieben ist, da der Medianlohn auf Basis von Lohngruppen berechnet wird und sich deswegen in 0,50 Euro Schritten verändert. Daher kann sich der tatsächliche Medianlohn in ostdeutschen Pflegeheimen um bis zu 0,49 Euro verändert haben, ohne eine Veränderung in dem auf Basis der Lohngruppen berechneten Medianlohn zu verursachen.

Tabelle 2 stellt die berechneten Eingriffsmaße dar. Neben den üblichen Eingriffsintensitätsmaßen wie dem Beschäftigtenanteil mit Stundenlöhnen unterhalb des Mindestlohns sowie dem Kaitz-Index wurde ebenfalls das von Machin et al. (2003) vorgeschlagene Maß der Lohnlücke berechnet. Dieses gibt an, um wie viel Prozent die Lohnsumme steigen muss, damit ceteris paribus alle Beschäftigten mindestens den Mindestlohn bekommen. Ähnlich wie bei der Interpretation des Medianlohns muss bei der Lohnlücke berücksichtigt werden, dass die Lohnlücke auf Basis von Lohngruppen in 0,50 Euro-Schritten berechnet wird. Da nicht bekannt ist, wie die Beschäftigten innerhalb dieser Lohngruppen verteilt sind, wurde zur Berechnung der Lohnsumme innerhalb der Gruppen eine Gleichverteilung angenommen. Für den Beschäftigtenanteil mit Stundenlöhnen unterhalb des Mindestlohns wurde das Maß sowohl ohne als auch mit Berücksichtigung der Mindestlohngruppe berechnet.

Die Eingriffsintensität unterscheidet sich sowohl zwischen den beiden Regionen mit unterschiedlichen Mindestlohnhöhen als auch nach der Art der Pflege. Grundsätzlich 
Tab. 2 Maße für die Eingriffsintensität

\begin{tabular}{|c|c|c|c|c|c|}
\hline \multirow[t]{2}{*}{$\mathrm{Maß}$} & & \multicolumn{2}{|l|}{ West } & \multicolumn{2}{|l|}{ Ost } \\
\hline & & Pflegedienste & Pflegeheime & Pflegedienste & Pflegeheime \\
\hline \multicolumn{6}{|l|}{ Ex Ante Betroffenheit } \\
\hline \multirow{2}{*}{$\begin{array}{l}\text { Anteil unterhalb des } \\
\text { Mindestlohns }\end{array}$} & Ohne ML-Lohngruppe & $4,8 \%$ & $5,8 \%$ & $6,2 \%$ & $4,3 \%$ \\
\hline & Mit ML-Lohngruppe & $8,9 \%$ & $10,5 \%$ & $28,4 \%$ & $17,6 \%$ \\
\hline Kaitz-Index & Medianlohn & $69,4 \%$ & $73,1 \%$ & $82,1 \%$ & $75,6 \%$ \\
\hline Lohnlücke & & $0,3 \%$ & $0,6 \%$ & $0,6 \%$ & $0,5 \%$ \\
\hline \multicolumn{6}{|l|}{ Ex Post Betroffenheit } \\
\hline \multirow{2}{*}{$\begin{array}{l}\text { Anteil unterhalb des } \\
\text { Mindestlohns }\end{array}$} & Ohne ML-Lohngruppe & $0,3 \%$ & $1,0 \%$ & $0,2 \%$ & $0,1 \%$ \\
\hline & Mit ML-Lohngruppe & $3,9 \%$ & $11,3 \%$ & $24,5 \%$ & $15,7 \%$ \\
\hline Kaitz-Index & Medianlohn & $67,1 \%$ & $72,9 \%$ & $80,6 \%$ & $75,9 \%$ \\
\hline
\end{tabular}

Quelle: Eigene Berechnungen auf Grundlage der T.I.P.-Pflegebefragung

ist die Eingriffsintensität in Ostdeutschland höher. Dabei ist der Unterschied bei Pflegeheimen nicht so groß wie bei Pflegediensten. Der Anteil der Beschäftigten in Pflegeheimen, die weniger als den Mindestlohn erhalten, liegt in Westdeutschland mit 5,8\% sogar über dem Anteil in Ostdeutschland $(4,3 \%)$, allerdings ist der Unterschied nicht signifikant. Wenig überraschend ist deshalb, dass die Stundenlöhne in westdeutschen Pflegeheimen ähnlich stark steigen müssen wie in Ostdeutschland. Die ausgewiesene Lohnlücke ergibt, dass wegen des Mindestlohns die Lohnsumme in westdeutschen Pflegeheimen ceteris paribus um 0,6\% steigen muss, damit alle Beschäftigten mindestens den Mindestlohn bekommen. Dieser Wert liegt etwas oberhalb der Lohnlücke in ostdeutschen Pflegeheimen $(0,5 \%)$. Auch hier ist der Unterschied nicht signifikant.

Aus den Lohnverteilungen ist ersichtlich, dass bereits vor der Mindestlohneinführung die Mindestlohngruppe in Ostdeutschland relativ gesehen deutlich mehr Bedeutung hat als in Westdeutschland. Die Lohnverteilung in Westdeutschland lässt den Modus der Lohnverteilung in der Lohngruppe 12,50-12,99 Euro für Pflegedienste und in der Lohngruppe 11,00-11,49 Euro für Pflegeheime vermuten, ${ }^{19}$ während der Modus in Ostdeutschland wie bereits erwähnt in beiden Bereichen in der Mindestlohngruppe liegt (Abb. 2). Folglich ist der Anteil der Beschäftigten, die vom Mindestlohn betroffen sind, in Ostdeutschland signifikant höher, wenn die Mindestlohngruppe mit berücksichtigt wird. ${ }^{20}$ Auch hier ist der Abstand bei den Pflegeheimen deutlich geringer als bei den Pflegediensten (Tab. 2).

\footnotetext{
${ }^{19}$ Dies gilt unter der Annahme, dass es keinen weiteren Anstieg der Lohnverteilung oberhalb von 14 Euro gibt. Auswertungen mit den IEB und der Verdienststrukturerhebung 2006 unterstützen diese Annahme.

${ }^{20}$ Sowohl für Pflegeheime als auch für Pflegedienste sind die Unterschiede in den Anteilen der Beschäftigten, die den Mindestlohn oder weniger verdienen, signifikant zum $1 \%$-Niveau.
}

In den qualitativen Untersuchungen wurde insbesondere durch die Fallstudien bestätigt, dass die Eingriffsintensität des Mindestlohns in Ostdeutschland höher war. Für diese Unterschiede wurden verschiedene Gründe angeführt. In Westdeutschland lagen nach Einschätzung der befragten Einrichtungen die Löhne bereits vor Einführung des Mindestlohns oberhalb des Mindestlohnniveaus. Unabhängig von der Trägerschaft wird in Westdeutschland oftmals nach Tarif bezahlt. Dies ist in Ostdeutschland häufig nicht der Fall. Eine Entlohnung unterhalb des Mindestlohns ist nach Ansicht einiger Befragter in westdeutschen Städten aufgrund der Arbeitsmarktlage nicht möglich. Diejenigen, die exakt den Mindestlohn erhalten, sind nach Einschätzung von Experten und Expertinnen (neugegründete Einrichtungen, Gewerkschaften, Verbände) die Minderheit. Davon liege der größte Teil im Bereich der privaten Pflegedienste. Allerdings wurde in den Expertengesprächen nicht ausgeschlossen, dass in einigen ländlichen und strukturschwachen Regionen in Westdeutschland unterhalb des Mindestlohnniveaus entlohnt wurde.

Die qualitativen Befunde (Fallstudien) zeigen auch, dass der Mindestlohn deutlich größere Auswirkungen auf Pflegehilfs- als auf Pflegefachkräfte hatte. Die Pflegefachkräfte bekamen in der Regel bereits wenigstens den Mindestlohn, während ein Teil der Pflegehilfskräfte erst durch die Einführung des Mindestlohns auf diesem Lohniveau entlohnt wurde. Der bestehende Fachkräftemangel sowie der für Pflegefach- und -hilfskräfte einheitliche Mindestlohn wurden als wesentliche Gründe für die geringe Eingriffsintensität bei Pflegefachkräften gesehen. Dabei wurde argumentiert, dass Pflegefachkräfte nicht geringer entlohnt werden können, da unter einem bestimmten Lohnniveau keine Fachkräfte zu bekommen seien. Pflegehilfskräfte sind daher eher als Begünstigte der Mindestlohneinführung anzusehen als Pflegefachkräfte. 
Die Unterschiede in der Höhe des Kaitz-Index lassen eine unterschiedliche Interpretation des Mindestlohns in Ostund Westdeutschland zu. Während der Kaitz-Index in Westdeutschland mit rund 70,0 \% des Medianlohns etwas oberhalb der zwei Drittel liegt, die für Definitionen des Niedriglohnbereichs verwendet werden (Kalina und Weinkopf 2010), sind die Werte für Ostdeutschland deutlich oberhalb dieses Niveaus. Der Mindestlohn in Ostdeutschland entspricht 75,6 \% des Medianlohns bei Pflegediensten und $82,1 \%$ bei Pflegeheimen und liegt damit in beiden Bereichen signifikant über den jeweiligen Werten in Westdeutschland. Die Höhe des Kaitz-Index spricht für eine vergleichsweise starke Eingriffsintensität des Mindestlohns in Ostdeutschland. $^{21}$

Auffällig an den ex post Maßen der Eingriffsintensität ist, dass in westdeutschen Pflegeheimen der Anteil der Beschäftigten, die den Mindestlohn oder weniger verdienen, gestiegen ist. Eine mögliche Erklärung für das beobachtete Phänomen kann aus den Fallstudien gewonnen werden. Hier gab es vereinzelt Hinweise, dass nach Einführung des Mindestlohns die Stundenlöhne von Beschäftigten, die etwas oberhalb des Mindestlohns verdient haben, auf das Mindestlohnniveau gesenkt wurden. Jedoch ist mit Hilfe der Betroffenheitsmaße nicht quantifizierbar, ob und wie stark dies den Anstieg der ex post Eingriffsintensität erklären kann.

\section{Ergebnisse}

Die Auswertungen der Betroffenheitsmaße lassen die Vermutung zu, dass die Mindestlohneinführung die Lohnhöhe verändert hat und geben damit auch Anlass zu einer Analyse der Beschäftigungswirkungen. Insbesondere in Ostdeutschland stellt der Mindestlohn einen starken Eingriff in die Lohnverteilung dar. Somit sind auch hier stärkere Effekte $\mathrm{zu}$ erwarten als in Westdeutschland. Im Folgenden wird zunächst auf das gewählte Modell, die verwendeten Variablen sowie die gewählte Stichprobe eingegangen. AnschlieBend werden die quantitativen und qualitativen Ergebnisse differenziert nach Lohneffekten und Beschäftigungswirkungen dargestellt. Abschließend werden noch Ergebnisse zu den Wettbewerbswirkungen aus den qualitativen Untersuchungsschritten beschrieben.

\subsection{Modellspezifikation}

Aufgrund der vorhandenen Datengrundlage ist die Auswahl der möglichen Schätzansätze stark begrenzt. Der vorliegende Paneldatensatz erfasst die Informationen auf Einrichtungsebene. Dies schließt den Einsatz von Schätzansätzen

\footnotetext{
${ }^{21}$ Für eine Diskussion der Höhe des Kaitz-Index siehe Rattenhuber (2011).
}

auf Individualebene aus. Neben dem hier gewählten Ansatz von Machin et al. (2003) kann ebenfalls ein DvD-Ansatz mit binärem Treatment gewählt werden, indem tarifgebundene Einrichtungen als Kontrollgruppe verwendet werden. Dieses Schätzverfahren wurde als Alternative in IAW (2011) durchgeführt. Allerdings wird das Verfahren von Machin et al. (2003) dem DvD-Ansatz vorgezogen, da die Tarifbindung sehr stark durch die Art des Trägers determiniert ist. Zudem gibt es keine Möglichkeiten, die Ergebnisse anhand von Vergleichsanalysen zu überprüfen.

Die T.I.P.-Pflegebefragung ermöglicht sowohl eine Berechnung des Wachstums der Durchschnittslöhne auf Ebene der Einrichtung als auch die Ermittlung der Veränderung der Beschäftigung. Wie bereits erwähnt, ist eine Berechnung der Durchschnittslöhne aufgrund der Rechtszensierung der Lohnverteilung bei 14 Euro schwierig. Verwendet man zur Berechnung für die Löhne oberhalb dieser Schwelle den Schwellenwert, impliziert dies, dass die Durchschnittslöhne tendenziell unterschätzt werden. Zwar ist die Lohnverteilung ebenfalls linkszensiert und bildet Löhne unter 6 Euro in einer Lohngruppe ab. Diese ist allerdings kaum besetzt, daher ist der Einfluss der Linkszensierung vernachlässigbar.

Das Problem kann reduziert werden, indem Einrichtungen mit relativ hohen Löhnen in den Schätzungen nicht berücksichtigt wurden. Da die Selektivitätsanalysen angedeutet haben, dass die Teilnahmebereitschaft bei weniger stark betroffenen Einrichtungen niedriger ist, wird zusätzlich die Teilnahmeselektion reduziert. Als Einrichtungen mit relativ hohen Löhnen wurden die Einrichtungen definiert, deren unterste Lohngruppe bei Pflegekräften vor Einführung des Mindestlohns mindestens 1,50 Euro oberhalb des jeweils gültigen Mindestlohns lag. ${ }^{22}$ In diesen Einrichtungen werden keine Lohneffekte aufgrund der Mindestlohneinführung erwartet.

Durch die Nichtberücksichtigung der Einrichtungen mit vergleichsweise hohen Löhnen fallen in Westdeutschland 135 und in Ostdeutschland 27 Einrichtungen aus der Stichprobe. Dementsprechend werden in Westdeutschland 119 Einrichtungen und in Ostdeutschland 120 Einrichtungen in den Schätzansätzen verwendet. Die Lohnverteilungen, insbesondere in Westdeutschland, zeigen, dass die Problematik der Rechtszensierung deutlich reduziert wird (Abb. 4). Zwar ist der Modus der Verteilung der reduzierten Stichprobe in Westdeutschland immer noch in der höchsten Lohngruppe, allerdings ist ein Großteil der rechtszensierten Stundenlöhne durch die Einschränkung nicht mehr in der Stichprobe enthalten.

Die Einrichtungen in der reduzierten Stichprobe sind sowohl in Westdeutschland als auch in Ostdeutschland etwas

\footnotetext{
${ }^{22}$ Es wurden auch Schätzungen mit dem kompletten Datensatz durchgeführt. Die Schätzungen für Ostdeutschland weisen kaum Unterschiede auf. In Westdeutschland sind die Koeffizienten der reduzierten Stichprobe deutlich kleiner (IAW 2011).
} 

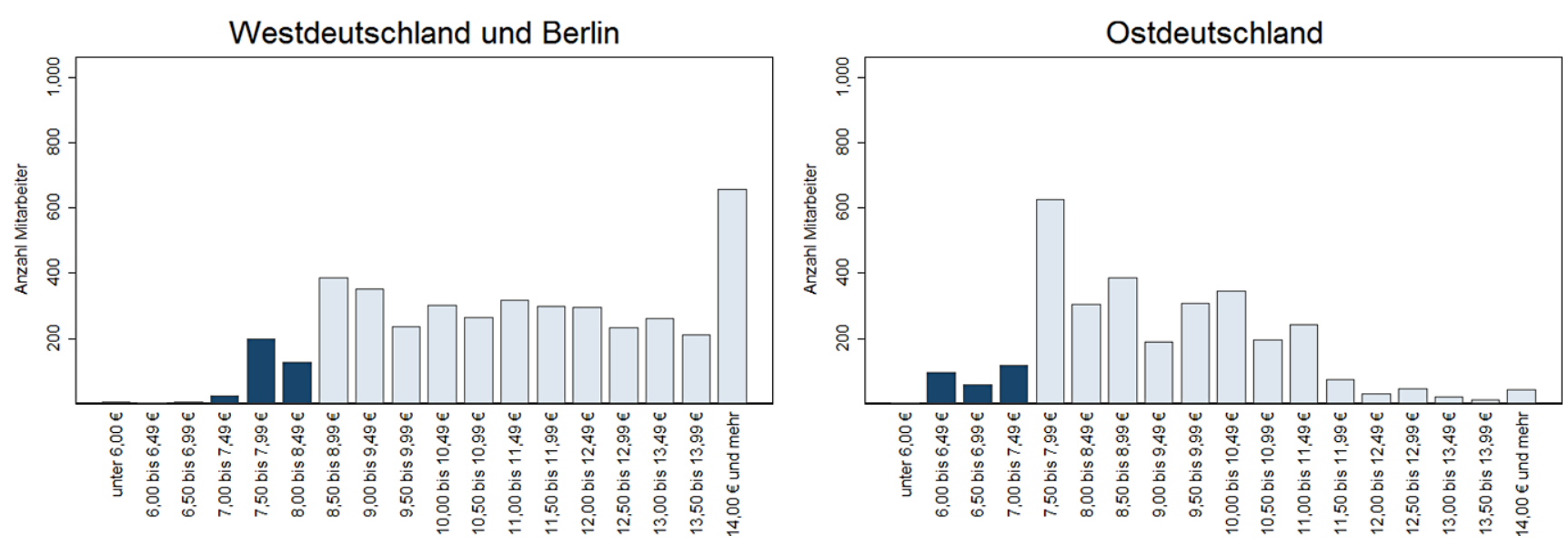

Abb. 4 Lohnverteilungen in der reduzierten Stichprobe. Anmerkung: Grundlage sind 254 Einrichtungen und 8.672 Beschäftigte in Westdeutschland sowie 147 Einrichtungen und 5.015 Beschäftigte in Ostdeutschland. Quelle: Eigene Berechnungen auf Grundlage der T.I.P.-Pflegebefragung

größer als die ausgeschlossenen Einrichtungen. In Westdeutschland sind in der reduzierten Stichprobe 38,5 Beschäftigte pro Einrichtung angestellt, während in den aus der Stichprobe ausgeschlossenen Einrichtungen 30,3 Beschäftigte pro Einrichtung arbeiten. Dieser Unterschied ist zum $10 \%$-Niveau signifikant. Unter den ostdeutschen Einrichtungen ist die durchschnittliche Beschäftigtenzahl mit 34,5 Beschäftigten pro Einrichtung in der reduzierten Stichprobe größer als bei den ausgeschlossenen Einrichtungen (32,3 Beschäftigte pro Einrichtung), allerdings ist der Unterschied nicht signifikant.

Durch die reduzierte Stichprobe befinden sich insbesondere weniger freigemeinnützige und kirchliche Einrichtungen in den Schätzungen. Der Anteil der freigemeinnützigen und kirchlichen Einrichtungen sinkt von 50,0 \% in Westdeutschland und 53,7\% in Ostdeutschland in der gesamten Stichprobe auf 40,9\% in West - beziehungsweise $45,8 \%$ in Ostdeutschland. Im Gegensatz dazu sind die privaten Einrichtungen deutlich stärker vertreten. In der gesamten Stichprobe sind 44,1\% der westdeutschen und 45,5\% der ostdeutschen Einrichtungen in privater Trägerschaft, während in der reduzierten Stichprobe die Anteile auf 52,1\% in Westdeutschland und 51,7 \% in Ostdeutschland steigen.

Als Ergebnisvariablen werden zwei unterschiedliche Maße verwendet. Der Einfluss des Mindestlohns auf die Lohnhöhe wird mit Hilfe der Veränderung des logarithmierten Durchschnittslohns gemessen. Der Einfluss der Beschäftigung wird über die Veränderung der logarithmierten Beschäftigung von Pflegekräften gemessen. Beide Ergebnisvariablen stellen demnach Veränderungsraten der beiden GröBen Durchschnittslohn und Beschäftigung dar.

Der Einfluss des Mindestlohns auf das Lohnwachstum der Durchschnittslöhne sowie auf das Beschäftigungswachstum wird mit Hilfe zweier Maße der Eingriffsintensität gemessen. Dies ist zum einen die von Machin et al. (2003) vorgeschlagene Lohnlücke als Maß für die finanzi- elle Betroffenheit der Einrichtungen sowie die personelle Betroffenheit, das heißt der Anteil der Beschäftigten, die direkt durch eine Lohnerhöhung vom Mindestlohn profitieren. Dabei muss bei der Interpretation der Ergebnisse zur Lohnlücke berücksichtigt werden, dass die exakten Stundenlöhne nicht bekannt sind und die Approximation mit dem Gruppenmittel Unschärfen enthält. Dies kann, abhängig von der tatsächlichen Lohnverteilung, innerhalb der Lohngruppen zu einer Unter- oder Überschätzung des Effekts der finanziellen Belastung auf das Wachstum des Durchschnittslohns und der Beschäftigung führen. Da das Maß der personellen Betroffenheit unabhängig von der Lohnhöhe der Beschäftigten berechnet wird, tritt dieses Problem beim Anteil der Beschäftigten mit Löhnen unterhalb des Mindestlohns nicht auf.

Die Effekte beider Treatmentvariablen wurden mit einer eng definierten Auswahl an Kovariaten sowie mit einem weiter gefassten Set an Kovariaten geschätzt. Die engere Definition der Kovariate umfasst ausschließlich Dummies für die Art der Pflege, das heißt, ob es sich um Pflegedienste oder -heime handelt, sowie für die Trägerart. ${ }^{23}$

\subsection{Lohneffekte}

Die Eingriffsintensitätsmaße zeigen, dass sich die Eingriffsintensität zwischen Pflegediensten und -heimen unterscheidet. Dies spricht für unterschiedliche Lohnstrukturen in den beiden Pflegebereichen und somit für unterschiedliche Lohnwachstumsraten. Aufgrund der unterschiedlichen Verbreitung kollektivrechtlicher Vereinbarungen sowie unterschiedlicher Organisationsformen in den verschiedenen Trägerformen ist eine Berücksichtigung der Trägerart in den

\footnotetext{
${ }^{23}$ Bei der Trägerart wird zwischen privaten, öffentlich-rechtlichen und freigemeinnützigen, inklusive der kirchlichen Einrichtungen, unterschieden.
} 
Tab. 3 Auswirkungen des Mindestlohns auf das Lohnwachstum

(1)

MA-anteil mit Löhnen < ML

Konstante

lvariablen

Beobachtungen

Korrigiertes $R^{\mathbf{2}}$

Ostdeutschland

Lohnlücke

MA-anteil mit Löhnen $<$ ML

Konstante

Kontrollvariablen

Beobachtungen

Korrigiertes $R^{2}$

\begin{tabular}{|c|c|c|c|c|}
\hline \multicolumn{5}{|l|}{ Westdeutschland und Berlin } \\
\hline Lohnlücke & $\begin{array}{l}1.0500^{* *} \\
(0.4650) \\
{[0.026]}\end{array}$ & $\begin{array}{l}1.0998^{* *} \\
(0.4507) \\
{[0.017]}\end{array}$ & & \\
\hline MA-anteil mit Löhnen $<$ ML & & & $\begin{array}{l}0.0864^{* *} \\
(0.0386) \\
{[0.027]}\end{array}$ & $\begin{array}{l}0.1220^{* * *} \\
(0.0401) \\
{[0.003]}\end{array}$ \\
\hline Konstante & $\begin{array}{l}0.0525 \\
(0.0386) \\
{[0.177]}\end{array}$ & $\begin{array}{l}0.0131 \\
(0.0749) \\
{[0.862]}\end{array}$ & $\begin{array}{l}0.0539 \\
(0.0386) \\
{[0.166]}\end{array}$ & $\begin{array}{l}0.0067 \\
(0.0737) \\
{[0.927]}\end{array}$ \\
\hline Kontrollvariablen & A & $\mathrm{A}, \mathrm{B}$ & A & $\mathrm{A}, \mathrm{B}$ \\
\hline Beobachtungen & 119 & 106 & 119 & 106 \\
\hline Korrigiertes $R^{2}$ & 0.017 & 0.083 & 0.016 & 0.113 \\
\hline \multicolumn{5}{|l|}{ Ostdeutschland } \\
\hline Lohnlücke & $\begin{array}{l}1.7177^{* * *} \\
(0.2981) \\
{[0.000]}\end{array}$ & $\begin{array}{l}1.6412^{* * *} \\
(0.2990) \\
{[0.000]}\end{array}$ & & \\
\hline MA-anteil mit Löhnen $<$ ML & & & $\begin{array}{l}0.1431^{* * *} \\
(0.0406) \\
{[0.001]}\end{array}$ & $\begin{array}{l}0.1712^{* * *} \\
(0.0391) \\
{[0.000]}\end{array}$ \\
\hline Konstante & $\begin{array}{l}-0.1556^{* * *} \\
(0.0503) \\
{[0.002]}\end{array}$ & $\begin{array}{l}-0.0720 \\
(0.0736) \\
{[0.331]}\end{array}$ & $\begin{array}{l}-0.1570^{* * *} \\
(0.0543) \\
{[0.005]}\end{array}$ & $\begin{array}{l}-0.0910 \\
(0.0770) \\
{[0.240]}\end{array}$ \\
\hline Kontrollvariablen & A & $\mathrm{A}, \mathrm{B}$ & A & A, B \\
\hline Beobachtungen & 120 & 105 & 120 & 105 \\
\hline Korrigiertes $R^{2}$ & 0.284 & 0.304 & 0.168 & 0.235 \\
\hline
\end{tabular}

(2)

(3)

(4)

Standardfehler in runden und $P$-Wert in eckigen Klammern. Kontrollvariablen: Set A: Dummies für Art der Pflege und Art des Trägers. Set B: Dummies für Tarifbindung, Unabhängige Lohngestaltung, Ertragslage sowie die Gesamtanzahl der Beschäftigten, Anteil Teilzeitbeschäftigter, Anteil Männer an der Beschäftigung sowie Anteil der Beschäftigten im persönlichen Geltungsbereich des Mindestlohnes

Signifikanzniveaus: ${ }^{*} 0,1,{ }^{* *} 0,05,{ }^{* * *} 0,01$

Schätzungen notwendig. ${ }^{24}$ Die unterschiedlichen Trägerformen wurden ebenso in der Auswahl der für die qualitativen Analysen befragten Einrichtungen berücksichtigt. Ergänzende Aspekte zu den quantitativen Untersuchungen konnten aus allen relevanten Teilbereichen der Pflegebranche in die Ergebnisse einfließen.

Aufgrund der unterschiedlichen Mindestlohnhöhen wurden alle Schätzungen separat für Ost- und Westdeutschland durchgeführt. Die Ergebnisse der Schätzungen sind in Tab. 3 dargestellt. Die Schätzergebnisse zeigen einen signifi-

\footnotetext{
${ }^{24}$ Alternativ könnten die Schätzungen separat für die beiden Pflegebereiche und die Trägerformen durchgeführt werden. Aufgrund der Fallzahlen lässt sich dies jedoch ausschließlich in Westdeutschland für die Unterscheidung nach Art der Pflege durchführen, wenn der vollständige Datensatz verwendet wird. Die Ergebnisse implizieren einen stärkeren Einfluss der Lohnlücke auf das Lohnwachstum bei Pflegediensten als bei Pflegeheimen. Im Unterschied dazu hat der Mitarbeiteranteil mit Stundenlöhnen unterhalb des Mindestlohns in Pflegeheimen einen stärkeren Effekt auf das Lohnwachstum.
}

kant positiven Einfluss der Mindestlohnmaße auf die Ergebnisvariable. In Westdeutschland erhöht eine um einen Prozentpunkt höhere Lohnlücke die Wachstumsrate des Durchschnittslohns um etwa 1,05 bis 1,10 Prozentpunkte. In Ostdeutschland führt eine um einen Prozentpunkt höhere Lohnlücke zu einer um 1,64 bis 1,72 Prozentpunkte höheren Wachstumsrate des Durchschnittslohns.

Auf den ersten Blick bestätigen die quantitativen Ergebnisse die Beobachtungen auf Basis der Eingriffsintensitäten. Die Koeffizienten in den Schätzungen für Ostdeutschland sind höher als in Westdeutschland allerdings ist der Unterschied nicht signifikant. Dementsprechend ist statistisch nicht nachweisbar, dass eine finanzielle Mehrbelastung durch den Mindestlohn in ostdeutschen Einrichtungen zu höheren Lohnwachstumsraten führt.

Ein ähnliches Bild zeigt sich bei der personellen Betroffenheit vom Mindestlohn. Ein um einen Prozentpunkt höherer Anteil der Beschäftigten mit Stundenlöhnen unterhalb des geltenden Mindestlohns erhöht die Wachstumsrate der Löhne in Westdeutschland um 0,09 bis 0,12 Prozentpunkte. 
Im Gegensatz dazu erhöht in Ostdeutschland ein um einen Prozentpunkt höherer Mitarbeiteranteil die Wachstumsrate des Durchschnittslohns um 0,14 bis 0,17 Prozentpunkte. Ebenso wie bei der Lohnlücke hat die personelle Betroffenheit vom Mindestlohn in Ostdeutschland einen stärkeren Anstieg der Durchschnittslöhne zur Folge als in Westdeutschland, allerdings sind die Unterschiede auch bei der personellen Betroffenheit nicht signifikant.

In den qualitativen Untersuchungen wurden Fragen zum Lohnniveau vor und nach der Mindestlohneinführung gestellt. Dazu liegen sowohl von einigen Experten und Expertinnen als auch von Leitern und Leiterinnen von Einrichtungen Erkenntnisse vor. Die Beschäftigten der Pflegeeinrichtungen konnten jeweils nur zu ihrer Person Auskunft über das Lohnverhältnis vor und nach Mindestlohneinführung geben, durften jedoch nicht über die Entlohnung anderer Mitarbeiter und Mitarbeiterinnen sprechen. Daher wurden die Beschäftigten - ebenso wie die Experten und Expertinnen - ergänzend um Einschätzungen allgemein zur Branche gebeten und gefragt, ob sich die Lohnstruktur verändert habe beziehungsweise ob es durch die Einführung des Mindestlohns zu Einsparungen des Personals gekommen sei (siehe auch Beschäftigungseffekte).

Insgesamt zeigen die qualitativen Ergebnisse, dass ein enger Zusammenhang zwischen der Eingriffsintensität und den Lohnveränderungen aufgrund des Mindestlohns besteht. Dabei wird deutlich, dass der Mindestlohn die Einrichtungen in Ostdeutschland stärker getroffen hat als in Westdeutschland. Viele westdeutsche Einrichtungen hatten ihre Pflegekräfte bereits vor Einführung des Mindestlohns nach TVöD bezahlt und somit über Mindestlohnniveau vergütet. Wenn Anpassungen aufgrund der Mindestlohneinführung vorgenommen wurden, dann haben diese vor allem die Pflegehilfskräfte betroffen, da Pflegefachkräfte in der Regel bereits vor Mindestlohneinführung auch aufgrund der angespannten Situation auf dem Arbeitsmarkt für Pflegefachkräfte deutlich besser verdient haben. Allerdings hatte der Mindestlohn indirekte Auswirkungen, da einige Einrichtungen nicht nur die Stundenlöhne der Pflegehilfskräfte angepasst haben, sondern die Stundenlöhne für Pflegefachkräfte gleichermaßen angehoben haben um den Lohnabstand zwischen Pflegefach- und Pflegehilfskräften beizubehalten.

Die meisten Kontrollvariablen der quantitativen Untersuchungen haben keine signifikanten Effekte auf die Lohnveränderung. Dabei ist auffällig, dass in Ostdeutschland die Art der Pflege keinen Einfluss auf die Lohnveränderung hat. Im Unterschied dazu haben in Ostdeutschland private sowie freigemeinnützige oder kirchliche Einrichtungen ein signifikant höheres Lohnwachstum als öffentlich-rechtliche Einrichtungen. Dies ist unter anderem dadurch zu erklären, dass öffentlich-rechtliche Einrichtungen an den Tarifvertrag des öffentlichen Dienstes (TVöD) gebunden sind. Zwar sind die meisten freigemeinnützigen und kirchlichen Einrichtungen ebenfalls an kollektivrechtliche Vereinbarungen gebunden, diese liegen aber teilweise deutlich unter den Regelungen im öffentlichen Dienst. Es ist kein Unterschied zwischen privaten und freigemeinnützigen oder kirchlichen Einrichtungen feststellbar. In Westdeutschland hat weder die Art der Pflege noch die Trägerart einen signifikanten Effekt.

Insgesamt deuten die quantitativen Ergebnisse auf einen starken Einfluss des Mindestlohns auf das Lohnwachstum des Durchschnittslohns hin. Allerdings ist zu beachten, dass das Schätzmodell eine Annahme trifft, die, soweit möglich, überprüft werden muss: Der Einfluss der Treatmentvariable auf die Ergebnisvariable darf ausschließlich auf das Treatment, in diesem Fall die Mindestlohneinführung, zurückgeführt werden. Dies trifft allerdings nur zu, wenn das Lohnwachstum von der Lohnhöhe nicht beeinflusst wird. Zur Überprüfung der Exogenitätsannahme können PlaceboExperimente als Robustheitsanalysen durchgeführt werden. Da der für die Schätzungen verwendete Datensatz ausschließlich den Mindestlohnzeitraum umfasst und bisher keine anderen Datensätze für den Zeitraum der Mindestlohneinführung existieren, können lediglich Vergleichsanalysen mit einem anderen Datensatz für eine Periode vor Mindestlohneinführung durchgeführt werden. Hierzu werden die IEB verwendet.

Im Unterschied zu der T.I.P.-Pflegebefragung handelt es sich bei den IEB um einen Personendatensatz und nicht um einen Betriebsdatensatz. Da in den durchgeführten Schätzansätzen ausschließlich Variablen auf Einrichtungsebene berücksichtigt wurden, werden die Angaben aus den IEB auf Einrichtungsebene aggregiert. Dies ist möglich, da die Stichprobenziehung auf Einrichtungsebene erfolgte. Dementsprechend werden die Vergleichsanalysen ebenso wie die eigentlichen Schätzansätze auf Einrichtungsebene und nicht auf individueller Ebene durchgeführt.

Im Unterschied zu den Befragungsdaten sind die Lohninformationen in den IEB als Tagesentgelte und nicht als Stundenlöhne enthalten. Für die Umsetzung des Schätzansatzes werden die Lohninformationen auf Stundenebene benötigt. Die dafür benötigte durchschnittliche normale Wochenarbeitszeit wurde im Mikrozensus für die Berufsgruppe „Altenpfleger im Sozialbereich“ berechnet. Dabei wurde die Wochenarbeitszeit differenziert nach Vollzeit, Teilzeit mit mehr als 20 Stunden die Woche, Teilzeit mit 20 Stunden oder weniger pro Woche sowie nach geringfügiger Beschäftigung.

Soweit möglich wurde eine ähnliche Spezifikation der Gleichung gewählt wie bei den eigentlichen Schätzungen. Allerdings sind Informationen wie Tarifbindung, Art der Trägerschaft, subjektive Ertragslage oder die Unabhängigkeit der Einrichtung in der Lohnsetzung in den IEB nicht verfügbar. Als Kontrollvariable wurde demnach für die Basisschätzung die Zugehörigkeit zum Wirtschaftszweig „Ambulante soziale Dienste (WZ03: 85.32.6)“ als Identifikator 
Tab. 4 Vergleichsanalysen für das Lohnwachstum mit den IEB (2008-2009)

\section{West}

Lohnlücke

MA-anteil mit Löhnen $<$ ML

Konstante

Kontrollvariablen

Beobachtungen

Korrigiertes $R^{2}$

Ost

Lohnlücke

Konstante

Kontrollvariablen

Beobachtungen

Korrigiertes $R^{2}$
(1)

\section{$0,5154^{* * *}$ \\ $(0,0174)$ \\ $[0,000]$}

(2)

(3)

$0,5303^{* * *}$

$(0,0171)$

$[0,000]$

$-0,0469^{* *}$
$(0,0215)$
$[0,029]$
$\mathrm{A}, \mathrm{B}$
2.035
0,387

$0,5480^{* * *}$
$(0,0278)$
$[0,000]$

$0,0475^{\text {***}}$
$(0,0050)$ [0,000]

A

2.035

0,353

$0,5691^{* * *}$
$(0,0257)$
$[0,000]$

$-0,002$

$(0,0047)$

[0,665]

A

953

0,354
0,0211

$(0,0251)$

[0,400]

A, B

953

0,365

$\begin{array}{ll}0,3500^{* * *} & 0,3918^{* * *} \\ (0,0142) & (0,0141) \\ {[0,000]} & {[0,000]} \\ -0,0008 & -0,0977^{* * *} \\ (0,0062) & (0,0224) \\ {[0,899]} & {[0,000]} \\ \mathrm{A} & \mathrm{A}, \mathrm{B} \\ 2.035 & 2.035 \\ 0,287 & 0,347\end{array}$

$\begin{array}{ll}0,1307^{* * *} & 0,1369^{* * *} \\ (0,0140) & (0,0153) \\ {[0,000]} & {[0,000]} \\ -0,0034 & 0,0166 \\ (0,0059) & (0,0289) \\ {[0,564]} & {[0,565]} \\ \text { A } & \text { A, B } \\ 953 & 953 \\ 0,103 & 0,174\end{array}$

Standardfehler in runden und $P$-Wert in eckigen Klammern. Kontrollvariablen: Set A: Dummy für Art der Pflege. Set B: Gesamtanzahl der Beschäftigten, Anzahl der Pflegekräfte, Anteil Frauen, Anteil Teilzeit

Signifikanzniveaus: ${ }^{*} 0,1,{ }^{* *} 0,05,{ }^{* * *} 0,01$

zwischen den beiden Arten der Pflege genommen. In der weitergehenden Spezifikation wurden die Gesamtanzahl der Beschäftigten, die Anzahl der Pflegekräfte, der Anteil weiblicher Beschäftigter sowie der Anteil der Teilzeitbeschäftigten als Kontrollvariablen integriert.

Als Schätzzeitpunkte wurden 2008, als Zeitpunkt der Stichprobenziehung, sowie 2009 als darauf folgende Periode gewählt. Der Abstand zwischen den beiden gewählten Zeitpunkten entspricht ungefähr dem Zeitabstand zwischen den beiden Befragungswellen. Die Ergebnisse der Vergleichsanalysen sind in Tab. 4 dargestellt.

Die Ergebnisse werfen Zweifel an der Exogenitätsannahme auf, da auch für den Zeitraum ohne Mindestlohneinführung ein signifikant positiver Effekt der Treatmentvariablen auf die Ergebnisvariable identifiziert werden kann. Dies lässt vermuten, dass die Ergebnisse der Schätzanalysen positiv verzerrt sind. Eine Erklärung dafür könnte sein, dass die Löhne in Unternehmen mit sehr niedrigen Ausgangslöhnen eine Tendenz haben, stärker zu steigen als in Unternehmen mit höheren Löhnen. Allerdings ist die Aussagekraft des Placebo-Experiments dadurch eingeschränkt, dass die
Datenbasis eine andere ist als in der Schätzung der Treatmenteffekte.

Auch Machin et al. (2003) identifizieren in ihren PlaceboExperimenten signifikant positive Effekte der Treatmentvariablen auf die Ergebnisvariable. Allerdings ergibt ein Vergleich der Koeffizienten, dass diese für den Zeitraum der Mindestlohneinführung signifikant höher waren. Ein direkter Vergleich der Koeffizienten, wie ihn Machin et al. (2003) vornehmen, kann in diesem Fall nicht durchgeführt werden, da die Schätzungen mit unterschiedlichen Datenquellen vorgenommen wurden. Allerdings sind die Koeffizienten der Vergleichsanalysen bei der finanziellen Betroffenheit in den Schätzungen für Ostdeutschland signifikant kleiner als in denen für Westdeutschland, während in den eigentlichen Schätzungen die Größenordnung umgekehrt ist, wenn auch insignifikant. Dies ist ein Indiz dafür, dass der in Ostdeutschland gemessene Effekt zumindest teilweise auf die Mindestlohneinführung zurückzuführen ist.

Die qualitativen Untersuchungen zeigen neben den vor$a b$ formulierten Leitfragen auch andere Aspekte im Zusammenhang mit der Mindestlohneinführung. Neben der Evi- 
denz für Lohnerhöhungen aufgrund der Mindestlohneinführung, gibt es auch Hinweise auf Lohnsenkungen. Es wurde von Beschäftigten berichtet, dass Stundenlöhne von Mitarbeitern und Mitarbeiterinnen, die etwas mehr als den Mindestlohnsatz verdienten, nach der Mindestlohneinführung auf Mindestlohnniveau abgesenkt wurden. Die Lohnabsenkungen führten dazu, dass Beschäftigte kündigten. Infolgedessen erhöhte sich die Arbeitsbelastung der verbleibenden Pflegekräfte deutlich. Darunter hatten neben den Pflegekräften vor allem die Pflegebedürftigen zu leiden, da sich die Qualität der Pflegeleistungen verschlechterte.

Es lässt sich nicht abschließend klären, ob die beschriebenen Lohnsenkungen lediglich in Einzelfällen auftraten oder ob diese bei manchen Trägern und in manchen Regionen systematisch aufgetreten sind. Vor dem Hintergrund des demographischen Wandels und zunehmenden Fachkräftemangels ist aber nicht anzunehmen, dass sich Lohnabsenkungen im Bereich der Pflegebranche halten können. Auch in den Expertengesprächen fand sich keine Bestätigung dieses Sachverhalts.

Von den Medizinischen Diensten der Krankenkassen wird ein Zusammenhang zwischen den gezahlten Löhnen und der Qualität der erbrachten Leistungen gesehen. Demnach erhöhen niedrige Löhne die Mitarbeiterfluktuation, insbesondere von Pflegefachkräften. Durch die hohe Fluktuation fällt es den betroffenen Einrichtungen schwer, die geforderten Qualitätsstandards einzuhalten. Inwieweit die Mindestlöhne die Fluktuation zu vermeiden helfen und damit zur Qualitätssicherung beitragen können, ist jedoch aufgrund der geringen Eingriffsintensität fraglich. Eine mögliche Verringerung der Fluktuation von Arbeitskräften als Ergebnis des Mindestlohns könnte allenfalls langfristig festgestellt werden.

Die Schätzungen ergeben, dass in Ostdeutschland eine Lohnerhöhung aufgrund der Mindestlohneinführung wahrscheinlich ist. Die Ergebnisse der qualitativen Untersuchungen bestätigen diesen Eindruck. Dies ist nicht weiter überraschend, da die Löhne in Ostdeutschland niedriger sind und die Eingriffsintensitätsmaße bereits Hinweise für eine stärkere Betroffenheit der ostdeutschen Einrichtungen gegeben haben. Dadurch könnte der Trend zu größeren Einrichtungen beschleunigt werden.

\subsection{Beschäftigungswirkungen}

Die Betroffenheitsmaße geben Anlass dazu, die Auswirkungen des Mindestlohns nicht nur auf die Lohnhöhe, sondern auch auf die Beschäftigung zu untersuchen. Ähnlich wie bei den Auswirkungen auf die Durchschnittslöhne werden ex ante stärkere Auswirkungen in Ostdeutschland vermutet, da die Einrichtungen hier stärker von der Mindestlohneinführung betroffen sind und dadurch auch stärkere Reaktionen zu erwarten sind.
Die Ergebnisse der Schätzungen in Tab. 5 zeigen weder für die finanzielle noch für die personelle Betroffenheit signifikante Effekte. Dies gilt sowohl für West- als auch für Ostdeutschland. In den qualitativen Untersuchungen wurden sowohl die Experten und Expertinnen als auch die Leitungen der Einrichtungen sowie die Beschäftigten danach gefragt, ob die Mindestlohneinführung Auswirkungen auf die Personalstruktur gehabt hätte und ob ein Personalabbau stattgefunden habe. Weder in den Fallstudien noch in den Expertengesprächen gab es Anzeichen für Beschäftigungseffekte.

Zur Überprüfung der Exogenitätsannahme wurden ana$\log$ zum Vorgehen bei den Schätzungen der Lohneffekte Vergleichsanalysen mit den IEB vorgenommen. Die Ergebnisse dieser Vergleichsanalysen in Tab. 6 weisen teilweise einen signifikant negativen Einfluss der Treatmentvariablen auf die Ergebnisvariablen auf, sind teilweise aber auch insignifikant. Dies zeigt, dass Einrichtungen mit einer geringen Entlohnung ein negatives Beschäftigungswachstum aufweisen.

Möglicherweise kann dies durch den Fachkräftemangel erklärt werden. Die qualitativen Analysen zeigen, dass es insbesondere in Westdeutschland schwierig ist, qualifizierte Pflegekräfte für den Mindestlohn zu finden. Einrichtungen, die ihren Pflegekräften weniger als den Mindestlohn zahlen, haben folglich Schwierigkeiten, ihr Personal zu halten beziehungsweise zu den gezahlten Löhnen neues Personal zu finden.

Der in Ost- und Westdeutschland unterschiedlich ausgeprägte Fachkräftemangel zeigt sich ebenfalls in den quantitativen Ergebnissen. Im Unterschied zu den Koeffizienten in den Vergleichsanalysen für Westdeutschland sind die Koeffizienten in den Vergleichsanalysen für Ostdeutschland größtenteils insignifikant. Dies spricht dafür, dass aufgrund der allgemeinen Arbeitsmarktlage die Auswirkungen des Fachkräftemangels in Ostdeutschland weniger stark sind. Eine mögliche Erklärung dafür wäre, dass sich in Ostdeutschland eine Umschulung zur Pflegekraft für Fachkräfte aus anderen Bereichen des Gesundheitsbereichs eher lohnt als in Westdeutschland.

Die insignifikanten Koeffizienten der Treatmentvariablen würden implizieren, dass der Mindestlohn keine Auswirkungen auf die Beschäftigung hätte. Allerdings sind die Koeffizienten für die Lohnlücke in den Vergleichsanalysen für Ostdeutschland negativ, während in den eigentlichen Schätzungen insignifikant positive Effekte identifiziert werden. Dies könnte ein Indiz für positive Beschäftigungseffekte in Ostdeutschland sein. Aus den Fallstudien ist bekannt, dass insbesondere kleinere ostdeutsche Einrichtungen von der Mindestlohneinführung betroffen waren. Durch eine stärkere Konzentration der Branche auf größere Einrichtungen sind Synergien denkbar, die bei großen Einrichtungen zu einem Beschäftigungszuwachs führen. Weiterhin könnten die Ergebnisse auf eine stärkere Marktmacht der Einrichtungen 
Tab. 5 Auswirkungen des Mindestlohns auf das Beschäftigungswachstum

\begin{tabular}{|c|c|c|c|c|}
\hline & \multicolumn{4}{|c|}{ Veränderung log Beschäftigung } \\
\hline & (1) & (2) & (3) & (4) \\
\hline \multicolumn{5}{|l|}{ West } \\
\hline Lohnlücke & $\begin{array}{l}-0.1777 \\
(2.8319) \\
{[0.950]}\end{array}$ & $\begin{array}{l}-1.8898 \\
(2.6111) \\
{[0.471]}\end{array}$ & & \\
\hline MA-anteil mit Löhnen < ML & & & $\begin{array}{l}-0.0290 \\
(0.2352) \\
{[0.902]}\end{array}$ & $\begin{array}{l}-0.1452 \\
(0.2364) \\
{[0.541]}\end{array}$ \\
\hline Konstante & $\begin{array}{l}-0.2303 \\
(0.2352) \\
{[0.330]}\end{array}$ & $\begin{array}{l}-0.4082 \\
(0.4336) \\
{[0.349]}\end{array}$ & $\begin{array}{l}-0.2303 \\
(0.2351) \\
{[0.329]}\end{array}$ & $\begin{array}{l}-0.4011 \\
(0.4342) \\
{[0.358]}\end{array}$ \\
\hline Kontrollvariablen & A & A, B & A & A, B \\
\hline Beobachtungen & 119 & 106 & 119 & 106 \\
\hline Korrigiertes $R^{2}$ & 0.000 & 0.185 & 0.000 & 0.184 \\
\hline \multicolumn{5}{|l|}{ Ost } \\
\hline Lohnlücke & $\begin{array}{l}1.3044 \\
(1.4702) \\
{[0.377]}\end{array}$ & $\begin{array}{l}2.4679 \\
(1.6589) \\
{[0.140]}\end{array}$ & & \\
\hline MA-anteil mit Löhnen < ML & & & $\begin{array}{l}0.1879 \\
(0.1854) \\
{[0.313]}\end{array}$ & $\begin{array}{l}0.3030 \\
(0.2072) \\
{[0.147]}\end{array}$ \\
\hline Konstante & $\begin{array}{l}-0.0479 \\
(0.2483) \\
{[0.847]}\end{array}$ & $\begin{array}{l}0.5089 \\
(0.4084) \\
{[0.216]}\end{array}$ & $\begin{array}{l}-0.0500 \\
(0.2480) \\
{[0.841]}\end{array}$ & $\begin{array}{l}0.4851 \\
(0.4075) \\
{[0.237]}\end{array}$ \\
\hline Kontrollvariablen & A & A, B & A & A, B \\
\hline Beobachtungen & 120 & 105 & 120 & 105 \\
\hline Korrigiertes $R^{2}$ & -0.013 & 0.044 & -0.011 & 0.043 \\
\hline
\end{tabular}

Standardfehler in runden und $P$-Wert in eckigen Klammern. Kontrollvariablen: Set A: Dummies für Art der Pflege und Art des Trägers. Set B: Dummies für Tarifbindung, Unabhängige Lohngestaltung, Ertragslage sowie die Gesamtanzahl der Beschäftigten, Anteil Teilzeitbeschäftigter, Anteil Männer an der Beschäftigung sowie Anteil der Beschäftigten im persönlichen Geltungsbereich des Mindestlohns

Signifikanzniveaus: ${ }^{*} 0,1,{ }^{* *} 0,05,{ }^{* * *} 0,01$

auf dem Arbeitsmarkt zurückzuführen sein. Allerdings lässt sich nicht eindeutig feststellen, ob die Beschäftigungseffekte des Mindestlohns in Ostdeutschland nicht vorhanden oder positiv sind, da hierfür ein direkter Vergleich der Koeffizienten notwendig wäre.

Auswertungen der T.I.P.-Pflegebefragung, der IEB und der Pflegestatistik haben ergeben, dass die Pflegebranche durch eine hohe Fluktuation der Beschäftigten gekennzeichnet ist. Teilweise kann dies auf Erfassungsprobleme zurückgeführt werden, allerdings konnte in den Expertengesprächen nicht abschließend geklärt werden, ob weitere Gründe für die Fluktuation verantwortlich sind. Aufgrund der teilweise signifikanten Ergebnisse in den Vergleichsanalysen (Tab. 6) ist nicht auszuschließen, dass niedrige Entlohnungen eine höhere Fluktuation verursachen. Da die Eingriffsintensitätsmaße höher sind, wenn entweder die Lohnlücke zum Mindestlohn wächst, oder wenn mehr Beschäftigte einen Stundenlohn unterhalb des Mindestlohns erhalten, ist es möglich, dass die Koeffizienten der Treatmentvaria- blen durch die Fluktuation in der Beschäftigung beeinflusst werden.

Ein möglicher Grund, warum keine Beschäftigungseffekte identifiziert werden können, ist der Fachkräftemangel. Grundsätzlich wird unabhängig vom Mindestlohn aufgrund des demographischen Wandels mit einem Beschäftigungswachstum in den nächsten Jahren gerechnet. Die Eingriffsintensität des Mindestlohns insgesamt wird dabei von den Expertinnen und Experten sowie in den Fallstudien als zu gering eingeschätzt, um zusätzliche Beschäftigungswirkungen hervorzurufen. Es bleibt allerdings abzuwarten, inwieweit sich der Fachkräftemangel so verschärfen wird, dass der Mindestlohn keine Relevanz mehr hat, da Pflegekräfte deutlich besser vergütet werden müssen.

Aufgrund der starken Regulierung der Pflegebranche können mögliche Substitutionseffekte zwischen Pflegehilfsund -fachkräften aufgrund des Mindestlohns nach Ansicht der Befragten ausgeschlossen werden. Eine Senkung der Personalkosten durch einen verstärkten Einsatz von Pflege- 
Tab. 6 Vergleichsanalysen für das Beschäftigungswachstum mit den IEB (2008-2009)

(1)

$-0.4075^{* *}$
$(0.1616)$
$[0.012]$

MA-anteil mit Löhnen $<$ ML

Konstante

Kontrollvariablen

Insgesamt

Korrigiertes $R^{2}$

Ost

Lohnlücke

MA-anteil mit Löhnen < ML

Konstante

Kontrollvariablen

Insgesamt

Korrigiertes $R^{2}$
2.036

0,005

$(0.0976)$
$[0.150]$

$0.0562^{* * *}$

$(0.0178)$

[0.002]

A

953

0,000
(2)

(3)

$-0.4803^{* * *}$

(0.1597)

[0.003]

$\begin{array}{ll}-0.4657^{* * *} & -0.5646^{* * *} \\ (0.1255) & (0.1267) \\ {[0.000]} & {[0.000]} \\ 0.1326^{* *} & 1.9303^{* * *} \\ (0.0545) & (0.2022) \\ {[0.015]} & {[0.000]} \\ \text { A } & \text { A, B } \\ 2.036 & 2.036 \\ 0,009 & 0,050\end{array}$

(4)
$1.8280^{* * *}$

$(0.2005)$

[0.000]

A, B

2.036

0,045

$-0.2210^{* *}$

(0.1060)

[0.037]

-0.0151
$(0.0512)$
$[0.769]$
0.0160
$(0.0969)$
$[0.869]$
A, B
953
0,001

Standardfehler in runden und $P$-Wert in eckigen Klammern. Kontrollvariablen: Set A: Dummy für Art der Pflege. Set B: Gesamtanzahl der Beschäftigten, Anzahl der Pflegekräfte, Anteil Frauen, Anteil Teilzeit

Signifikanzniveaus: ${ }^{*} 0,1,{ }^{* *} 0,05,{ }^{* * *} 0,01$

hilfskräften anstatt von Pflegefachkräften kann lediglich in eingeschränktem Maß geschehen, da gesetzlich eine Fachkraftquote von $50 \%$ vorgeschrieben ist und die meisten Einrichtungen zur Minimierung der Personalkosten bereits vor der Mindestlohneinführung diese Quote genau einhalten. ${ }^{25}$ Zudem wird bezweifelt, dass der Mindestlohn zu einer Substitution der teurer gewordenen Pflegehilfskräfte durch besser ausgebildete Pflegefachkräfte geführt hat, da durch den Fachkräftemangel Einrichtungen bereits Schwierigkeiten haben, qualifizierte Fachkräfte für ihre freien Stellen zu bekommen. Vorstellbar ist nach Aussage mancher Expertinnen und Experten allerdings, dass der Mindestlohn zur Reorganisationen der Arbeitsabläufe geführt hat, so dass bestimmte Tätigkeiten, die nicht zwingend durch Pflegekräfte durchgeführt werden müssen, von Hauswirtschaftskräften erledigt werden.

Eine weitere Möglichkeit, den Mindestlohn zu umgehen, ist der verstärkte Einsatz von Selbstständigen. Diese unter-

\footnotetext{
${ }^{25}$ Aufgrund des Fachkräftemangels prognostiziert ein Arbeitnehmervertreter eine Reduktion der gesetzlichen Fachkraftquote.
}

liegen nicht den Mindestlohnbestimmungen, da diese ausschließlich auf abhängig Beschäftigte Anwendung finden. Nach der qualitativen Evidenz ist in der Pflegebranche ein Trend zur Substitution von Beschäftigten durch Selbstständige zu beobachten. Oftmals sind diese zu $50 \%$ in einer Einrichtung angestellt und arbeiten zu $50 \%$ selbstständig. Dieser Trend existierte allerdings unabhängig von der Mindestlohneinführung bereits seit ein paar Jahren. Auch nach Beobachtungen von Vertreterinnen und Vertretern der Finanzkontrolle Schwarzarbeit sowie der Hauptzollämter ist keine verstärkte Substitution durch Selbständige infolge des Mindestlohns erfolgt. Da die Mindestlohneinführung jedoch erst zum 1. August 2010 eintrat, muss der Verlauf der Entwicklung abgewartet werden, um abschließend bewerten zu können, inwieweit die Mindestlohneinführung diesen Trend verstärkt hat.

Ebenfalls wurden in den qualitativen Interviews mögliche Auswirkungen des Mindestlohns vor dem Hintergrund der Ausweitung der allgemeinen Freizügigkeit für Arbeitnehmer und Arbeitnehmerinnen auf einen Großteil der osteuropäischen EU-Mitgliedsländer seit dem 1. Mai 2011 the- 
matisiert. Fragen zu diesem Themenbereich wurden Gewerkschaften, Verbänden, der Finanzkontrolle Schwarzarbeit sowie den Hauptzollämtern und der Zentrale Auslandsund Fachvermittlung gestellt. Ebenso wurden in den Einrichtungen sowohl die Geschäftsleitungen als auch die Pflegekräfte hierzu interviewt. Ein Großteil der Expertinnen und Experten konnte aufgrund der kurzen Frist seit Einführung der Ausweitung der Arbeitnehmerfreizügigkeit noch keine fundierten Aussagen treffen. Generell war man der Ansicht, dass der Mindestlohn eine Abgrenzung nach unten biete. Es wurde jedoch bemerkt, dass ausländische Arbeitnehmer und Arbeitnehmerinnen teilweise Probleme hätten, von ihrem ausländischen Arbeitgeber tatsächlich mit dem Mindestlohn entlohnt zu werden. Es herrschte weitestgehend Einigkeit darüber, dass kein Ansturm ausländischer Fachkräfte zu erwarten sei, da zum einen Deutschland kein attraktives Ziel sei und zum anderen bereits vorher viele ausländische Arbeitnehmer und Arbeitnehmerinnen in Deutschland gewesen wären. Diese Ansicht teilten auch alle befragten Einrichtungen. Ein Grund wird unter anderem auch darin gesehen, dass auf dem deutschen Pflegemarkt ein hoher Dokumentationsaufwand (bis zu 30-40 \% der Arbeitszeit) gefordert werde, der von vielen ausländischen Arbeitnehmern und Arbeitnehmerinnen aufgrund sprachlicher Barrieren nicht geleistet werden könne. Die Zentrale Auslandsund Fachvermittlung konnte ebenfalls keine Veränderungen des Fachkräfteangebots seit Einführung der Arbeitnehmerfreizügigkeit beobachten. Vertreter und Vertreterinnen der Finanzkontrolle Schwarzarbeit konnten keine spezifischen Aussagen zur Pflegebranche treffen, allerdings habe sich der in einem ostdeutschen Hauptzollamt befürchtete Ansturm von ausländischen Fachkräften bislang noch nicht eingestellt (Stand 31. August 2011).

\subsection{Wettbewerbswirkungen}

Abhängig von der Höhe der Entlohnung vor Einführung des Mindestlohns sowie insbesondere vom Lohnniveau in der Region wird der Einfluss des Mindestlohns auf die Wettbewerbsposition der betroffenen Unternehmen unterschiedlich bewertet. Dies ist nicht weiter überraschend, da in den Fallstudien angegeben wurde, dass die Personalkosten in der Pflegebranche etwa 70-90 \% der Gesamtkosten ausmachen. Dadurch tritt mit einer durch den Mindestlohn bedingten Erhöhung der Lohnkosten eine Verschlechterung der Wettbewerbsposition im Verhältnis zum Zeitpunkt vor der Einführung des Mindestlohns ein. Dementsprechend werden die Wettbewerbswirkungen in Ostdeutschland erheblich stärker gesehen, da hier das Lohnniveau in der Regel deutlich niedriger ist als in Westdeutschland.

In Ostdeutschland werden Auswirkungen der Mindestlohneinführung vor allem auf kleinere Einrichtungen vermutet. Größere Einrichtungen haben oft bereits vor der Ein- führung höhere Löhne gezahlt. Durch die Mindestlohneinführung werden größere Einrichtungen wettbewerbsfähiger, während die kleineren Einrichtungen teilweise Probleme haben, mit den höheren Löhnen zu wirtschaften. Die Konzentration der Branche hat bereits, wie in der Pflegestatistik zu beobachten ist, vor Mindestlohneinführung eingesetzt. Inwieweit dieser Trend durch den Mindestlohn beschleunigt wurde, geben die Expertengespräche nicht eindeutig zu erkennen.

In Regionen mit einem ohnehin bereits hohen Lohnniveau werden kaum Wettbewerbseffekte wahrgenommen. Aufgrund des Fachkräftemangels mussten Einrichtungen bereits vor der Mindestlohneinführung teilweise deutlich höhere Löhne zahlen, um geeignetes Personal zu finden.

\section{Bewertung des Mindestlohns durch die Betroffenen}

Ein interessanter Aspekt, der weder in den amtlichen Daten noch in der Pflegestatistik erhoben wurde, ist die Beurteilung des Mindestlohns durch die betroffenen Einrichtungen, die Pflegekräfte sowie die Expertinnen und Experten, da hierdurch die unterschiedlichen Perspektiven und insbesondere auch die Ansichten der Befragten dargestellt werden können. In den qualitativen Untersuchungen fand eine solche Beurteilung der Einführung sowie der Höhe des Mindestlohns statt.

Die Einführung des Mindestlohns wurde von den meisten Befragten als notwendig empfunden, um eine Lohnuntergrenze zu sichern, die den Lohnwettbewerb beschränkt oder Substitution durch günstigere ausländische Fachkräfte verhindert.

Die Geschäftsführungen von Einrichtungen, die eine geringe Eingriffsintensität aufweisen, bewerteten im Vergleich zu Geschäftsführungen von Einrichtungen mit einer hohen Eingriffsintensität die Höhe des Mindestlohns als zu niedrig. Dementsprechend waren die Forderungen nach einem höheren Mindestlohn in der Pflegebranche tendenziell den Einrichtungen zuzuordnen, die bereits vor Einführung des Mindestlohns über Mindestlohnniveau entlohnt hatten.

Weniger differenziert fiel die Beurteilung der Pflegekräfte aus. Diese hielten die Höhe des Mindestlohns in der Regel für zu niedrig. Sie forderten ein Mindestlohnniveau, das die Deckung der Lebenshaltungskosten ermöglicht. Ein hoher Anteil von Teilzeitkräften in der Pflegebranche verschärft diese Problematik zusätzlich. Die Expertinnen und Experten sahen den Mindestlohn in der Pflegebranche teils als zu niedrig an, teils wurde der Mindestlohn im Sinne eines Einstiegslohns oder einer absoluten Lohnuntergrenze für ausreichend gehalten. Dabei sahen neugegründete Einrichtungen sowie Gewerkschaften den Mindestlohn tendenziell als zu niedrig an, Dienstgeber in der Tendenz als ausreichend. Ein höherer Mindestlohn könnte, nach Einschätzung der Expertinnen und Experten, die Preisentwicklung 
in der Pflegebranche beeinflussen und Pflegeleistungen verteuern.

Neben der Kritik an der allgemeinen Höhe des Mindestlohns wurde die Differenzierung der Mindestlohnhöhe nach Ost- und Westdeutschland - insbesondere von Pflegefachund Pflegehilfskräften - kritisiert. Die Gesprächspartnerinnen und -partner sprachen sich für einen einheitlichen Mindestlohn aus. Diese Ansicht vertraten sowohl Einrichtungen aus Ost- als auch aus Westdeutschland. Statt einer regionalen Differenzierung der Mindestlöhne wurde oftmals ein differenzierter Mindestlohn für Pflegehilfs- und Pflegefachkräfte vorgeschlagen.

\section{Schlussfolgerungen und Interpretation}

Die Mindestlohneinführung in der Pflegebranche stellt einige Herausforderungen an eine Evaluation. Diese liegen zum Teil in den Besonderheiten der Pflegebranche, wie beispielsweise den zwei sehr unterschiedlichen Bereichen Pflegedienste und Pflegeheime, der heterogenen Trägerlandschaft oder der Abhängigkeit von der demographischen Entwicklung. Ein weiterer Aspekt, der eine Evaluation erschwert, liegt am Zeitpunkt der Mindestlohneinführung. Da der Mindestlohn in der Pflegebranche erst seit August 2010 existiert, fehlen bislang amtliche Daten für den Zeitraum nach der Mindestlohneinführung. Allerdings ermöglicht die erst kurz vor der Studie erfolgte Einführung des Mindestlohns eine genauere Einschätzung und Bewertung des Mindestlohns durch die Beteiligten. Zudem besteht die Möglichkeit mit einer Befragung einen Datensatz zu erstellen, der die benötigten Merkmale, wie zum Beispiel Stundenlöhne, enthält. Außerdem können Einschätzungen und Einstellungen der Betroffenen in den Analysen berücksichtigt werden.

Wie die Jahre vor der Mindestlohneinführung gezeigt haben, handelt es sich bei der Pflegebranche um eine, unabhängig von der Konjunkturentwicklung, kontinuierlich wachsende Branche. Dies äußert sich auch in einem Fachkräftemangel, der es den Einrichtungen erschwert, geeignetes Personal zu finden. In der Regel sollte diese Situation, unabhängig von einem Mindestlohn, zu höheren Löhnen und einer verbesserten Arbeitssituation führen. Abhängig von der Mindestlohnhöhe hat dieser entweder kaum bis gar keine Auswirkungen auf die Lohnhöhe, da die Wettbewerbssituation bereits die Zahlung höherer Löhne erfordert, oder sie beschleunigt die Anpassung der Marktlöhne an den Gleichgewichtslohn.

Die Maße der Eingriffsintensität sowie die Erkenntnisse aus den Expertengesprächen und Fallstudien deuten auf eine unterschiedliche Situation in West- und Ostdeutschland hin. Die Eingriffsintensitätsmaße lassen eine stärkere Belastung ostdeutscher Einrichtungen durch den Mindestlohn vermuten. Auch in den Schätzungen zu den Lohneffekten spiegelt sich dies wider, die in Ostdeutschland auf eine Erhöhung der Durchschnittslöhne aufgrund der Mindestlohneinführung hindeuten. Dies trifft sowohl auf Einrichtungen, die eine besonders hohe finanzielle Betroffenheit aufweisen, als auch auf Einrichtungen mit einer hohen personellen Betroffenheit zu. Zudem haben im Wesentlichen Pflegehilfskräfte von der Mindestlohneinführung profitiert, da durch den akuten Fachkräftemangel die Stundenlöhne von Pflegefachkräften insbesondere in Westdeutschland bereits vor Einführung des Mindestlohns deutlich oberhalb des Mindestlohns lagen.

Weder quantitativ noch qualitativ konnten Beschäftigungseffekte identifiziert werden. Inwieweit der Mindestlohn tatsächlich keine Auswirkungen auf die Beschäftigung hatte oder ob dieser Effekt darauf zurückzuführen ist, dass die Exogenitätsannahme bei den verwendeten Treatmentvariablen nicht hält, lässt sich aufgrund der fehlenden Vergleichbarkeit der Schätzergebnisse nicht abschließend sagen. Allerdings deuten die Ergebnisse aus den Expertengesprächen und Fallstudien darauf hin, dass der Mindestlohn keine Auswirkungen auf die Beschäftigung hatte. insbesondere die Tatsache, dass die Pflegebranche ein Wachstumsmarkt mit einem sich verschärfenden Fachkräftemangel ist, wird dafür verantwortlich gemacht.

Grundsätzlich sind aus den Expertengesprächen und Fallstudien zwei Trends in der Pflegebranche auszumachen. Zum einen findet eine Konzentration der Branche zu gröBeren Einrichtungen statt, zum anderen nimmt die Anzahl der Pflegekräfte, die bei einer Einrichtung teilzeitbeschäftigt sind und neben dieser Teilzeitbeschäftigung selbstständig als Pflegekräfte tätig sind, zu. Beide Trends waren bereits vor Mindestlohneinführung zu beobachten. Jedoch könnte die Mindestlohneinführung diese Trends verstärkt haben. Insbesondere die Konzentration auf größere Einrichtungen wird in einigen ostdeutschen Regionen direkt im Zusammenhang mit der Mindestlohneinführung gesehen, da der Pflegebereich nach SGB XI aufgrund der höheren Personalkosten für kleinere Anbieter nicht mehr attraktiv genug sei.

Dementsprechend werden in Ostdeutschland die Auswirkungen des Mindestlohns auf den Wettbewerb sehr viel stärker wahrgenommen als in Westdeutschland. Die Ergebnisse zeigen, dass in Westdeutschland der Mindestlohn kein geeignetes Mittel zur Lösung der Probleme auf dem Arbeitsmarkt für Pflegekräfte ist, da der Wettbewerbslohn auf dem Arbeitsmarkt deutlich oberhalb des Mindestlohnniveaus liegt. Folglich kann in Ostdeutschland der Mindestlohn durchaus als ein adäquates Mittel zu einer Verbesserung der Situation der Pflegekräfte angesehen werden, während dies auf Westdeutschland aufgrund der im Vergleich zu den bereits gezahlten Löhnen relativ geringen Mindestlohnhöhe nicht zutrifft. 
Sehr ambivalent fiel hingegen die Beurteilung des Mindestlohns durch die Betroffenen aus. Einerseits wurde der Mindestlohn begrüßt, da dieser eine Beschäftigung zu sehr niedrigen Stundenlöhnen unterbindet. Andererseits kritisierten insbesondere viele Pflegehilfskräfte, dass der Mindestlohn zu niedrig sei, da dieser die Lebenshaltungskosten nicht decken würde. Durch den hohen Anteil an Teilzeitbeschäftigten wird dieses Problem weiter verschärft. Gleichzeitig wurde auch kritisiert, dass der Mindestlohn in Ostdeutschland niedriger ist als in Westdeutschland. Hier wird ein einheitlicher Mindestlohn befürwortet.

Zusammenfassend kann festgehalten werden, dass der Mindestlohn in Ostdeutschland zu einer Anhebung der Löhne geführt hat, während Beschäftigungseffekte weder empirisch nachgewiesen werden können noch von den befragten Expertinnen und Experten erwartet werden. Allerdings beziehen sich die Erkenntnisse ausschließlich auf kurzfristige Effekte, da für mittel- bis langfristige Effekte der Zeitraum nach Mindestlohneinführung noch zu kurz war. Für eine empirische Untersuchung der mittel- bis langfristigen Auswirkungen der Mindestlöhne sollte zudem abgewartet werden, bis amtliche Daten den Zeitraum nach der Mindestlohneinführung in ausreichendem Umfang erfassen.

\section{Kurzfassung}

Die Pflegebranche ist mit 760.577 sozialversicherungspflichtig Beschäftigten eine der größten Branchen und ist in den letzten Jahren konstant gewachsen. Daher ist mit einem zunehmenden Fachkräftebedarf zu rechnen. Sie ist die einzige Branche des Gesundheitsbereichs mit einem Mindestlohn nach dem Arbeitnehmerentsendegesetz (AEntG). Da aufgrund der heterogenen Trägerlandschaft in der Pflegebranche kein nach § 7 AEntG repräsentativer Tarifvertrag existiert, wurde 2009 bei der Erneuerung des AEntG ein eigener Abschnitt für die Pflegebranche integriert, die eine Mindestlohnfestlegung durch eine achtköpfige Kommission festlegt.

Im Rahmen der Studie sollen folgende Fragen beantwortet werden: Inwieweit werden die Besonderheiten der Branche bei der Umsetzung des Mindestlohns berücksichtigt? Welche Auswirkungen sind durch einen Mindestlohn in einer Wachstumsbranche mit zunehmendem Fachkräftemangel zu erwarten und empirisch festzustellen? Ist die Festsetzung eines Mindestlohns das geeignete Mittel, um die Probleme auf dem Arbeitsmarkt für Pflegeleistungen zu lösen?

Zur Beantwortung dieser Fragen werden sowohl qualitative als auch quantitative Methoden der Wirtschafts- und Sozialforschung angewandt. Für die qualitativen Analysen wurde eine Kombination des Fallstudienkonzepts von Apel (2009) und der Grounded Theory von Strauss (Lamnek
2005) verwendet. Dies ermöglicht eine genauere und umfassendere Darstellung der Sachverhalte. Für die quantitativen Analysen wurde auf ein Schätzmodell von Machin et al. (2003) zurückgegriffen, was kontinuierliche Maße als Treatment verwendet und die gleichen Untersuchungseinheiten über den Zeitraum der Mindestlohneinführung hinweg betrachtet.

Als Grundlage für die Schätzungen und die deskriptiven Auswertungen dient eine assistierte Online-befragung in zwei Wellen, die den Zeitraum um die Mindestlohneinführung umfassen. Ergänzt werden die Auswertungen der Befragungsdaten durch amtliche Statistiken.

Die Auswertungen wurden für die zwei unterschiedlichen Bereiche der Pflegebranche, der ambulanten und der stationären Pflege, durchgeführt. Die Ergebnisse zeigen, dass trotz eines konstanten Wachstums der Branche zwischen 2003 und 2009, die Einkommen der Beschäftigten in der Branche nicht gestiegen sind. Dabei ist gleichzeitig eine Konzentration der Branche zu größeren Einrichtungen zu beobachten. Diese Entwicklung führt zu einer Verschärfung des Wettbewerbs um Arbeitskräfte, der aber nur eingeschränkt über die Löhne ausgetragen werden kann, da der Absatzmarkt von Pflegeleistungen monopsonähnliche Strukturen aufweist. Dies zeigt sich unter anderem daran, dass die Pflegesätze zwischen 2003 und 2009 geringer angestiegen sind als die jährliche Inflationsrate.

Sowohl die Maße für die Eingriffsintensität des Mindestlohns als auch die Ergebnisse der qualitativen Analysen deuten darauf hin, dass Ostdeutschland stärker vom Mindestlohn betroffen ist als Westdeutschland und Berlin. Dies liegt vor allem daran, dass, wie auch die qualitativen Untersuchungen bestätigen, das Lohnniveau in Westdeutschland und Berlin bereits vor Mindestlohneinführung in der Regel deutlich oberhalb des Mindestlohns lag.

Für die Schätzungen der Effekte des Mindestlohns auf die Lohnhöhe sowie die Beschäftigung werden zwei unterschiedliche Treatmentvariablen verwendet. Das erste Maß spiegelt die finanzielle Betroffenheit der Einrichtungen wider, da es misst um wie viel die Lohnsumme ceteris paribus steigen muss, damit alle Beschäftigten mindestens den Mindestlohn bekommen. Als zweites Maß wird der Anteil der Beschäftigten, die vor Mindestlohneinführung weniger als den Mindestlohn bekommen haben, verwendet. Dieses Maß gibt die personelle Betroffenheit der Einrichtungen wieder.

Die Ergebnisse der quantitativen Analysen zeigen einen signifikant positiven Effekt des Mindestlohns auf das Lohnwachstum. Dies gilt sowohl für die finanzielle als auch die personelle Betroffenheit. Allerdings zeigen Vergleichsanalysen, dass dieser Effekt zumindest teilweise auf eine vom Mindestlohn unabhängige Entwicklung der Löhne zurückzuführen ist. Die Ergebnisse legen jedoch nahe, dass die Einführung des Mindestlohns einen positiven Effekt auf das Lohnwachstum in ostdeutschen Einrichtungen hatte. Dies wird durch die qualitativen Untersuchungen bestätigt. 
Im Unterschied zu den Ergebnissen für die Lohnhöhe können keine signifikanten Effekte der Treatmentvariablen auf die Beschäftigungsentwicklung gefunden werden. Die Vergleichsanalysen identifizieren jedoch einen signifikant negativen Effekt der finanziellen Betroffenheit auf das Beschäftigungswachstum. Wie Analysen der Pflegestatistik zeigen, ist die Branche durch eine sehr starke Fluktuation der Beschäftigten gekennzeichnet. Die signifikant negativen Ergebnisse der Vergleichsanalysen könnten ein Hinweis darauf sein, dass die Fluktuation der Beschäftigten in Einrichtungen mit einer niedrigen Entlohnung höher ist und dadurch die Treatmentvariable beeinflusst wird.

In den Fallstudien und Expertengesprächen wurde ein Einfluss des Mindestlohns auf die Beschäftigung bezweifelt. Durch den akuten Fachkräftemangel wird unabhängig vom Mindestlohn mit einem Beschäftigungswachstum gerechnet, da aufgrund des demographischen Wandels die Nachfrage nach Pflegeleistungen steigt.

Die Auswirkungen des Mindestlohns auf den Wettbewerb werden sehr unterschiedlich wahrgenommen. Insbesondere in Ostdeutschland scheinen kleinere Einrichtungen von der Mindestlohneinführung betroffen gewesen zu sein, die dadurch ihre Wettbewerbsfähigkeit gegenüber größeren Konkurrenten verloren haben. Gleichzeitig ist die Forderung von den Einrichtungen, die bereits vor der Mindestlohneinführung oberhalb des Mindestlohns entlohnt haben, nach einem höheren Mindestlohn ein Indiz, dass mit dem Mindestlohn ein Wettbewerb über die Löhne verhindert werden soll.

Von den meisten Gesprächspartnern in den qualitativen Analysen wurde die Mindestlohneinführung begrüßt. Teilweise wurde die Umsetzung, beispielsweise die unterschiedlichen Lohnhöhen in Ost- und Westdeutschland sowie keine Differenzierung in der Lohnhöhe zwischen Pflegefach- und Pflegehilfskräften, kritisiert.

\section{Executive summary}

The care sector is one of the largest in the German economy. It employs 760,577 workers who are subject to social insurance contribution. Within the last years, the sector has increased continually leading to a growing demand for qualified carers for the elderly. It is the only industry in the health sector with a minimum wage, according to the Posted Workers Act (AEntG). Due to the different organisational institutions in the care sector, there is no representative collective agreement, which according to $\S 7$ AentG is a necessary condition for a sectorial minimum wage. Therefore, a separate section was added to the AEntG in 2009 enabling for the introduction of a minimum wage specifically for the care sector. According to this section, a commission of eight representatives of employers and employees in the care sector set a minimum wage.
The study tries to give answers to the following questions: To what extent have the characteristics of the care sector been taken into account during the implementation of the minimum wage? What is the expected and empirically observable impact of a minimum wage on a growing industry with an increasing shortage of skilled workers? Is a minimum wage the appropriate way to solve the problems in the labour market for nursing services?

To answer these questions, we applied both qualitative and quantitative methods of economic and social research. For the qualitative analyses, we used a combination of the case study approach by Apel (2009) and the Grounded Theory by Strauss (Lamnek 2005). This approach allows for a more accurate and comprehensive representation of the facts. The quantitative analyses are based on an estimation model by Machin et al. (2003), which uses continuous measures as treatment and considers the same units over the period when the minimum wage was introduced.

An assisted online survey in two waves of care sector units was used for most of the estimations and descriptive analyses. The survey covered the introduction of the minimum wage by collecting information on the status before the minimum wage introduction in the first wave and on the status afterwards in the second wave.

The analyses were performed separately for the two areas of the care sector: The operation of nursing homes for the elderly and the provision of domestic nursing services. The results show that despite a steady growth of the sector between 2003 and 2009, carers' incomes have not increased. Furthermore, a concentration to larger facilities can be observed. This development leads to an increased competition for labour, which has not yet affected wages, because the market for nursing services is close to a monopsony. Therefore, the prices for nursing services have increased to a lesser extent than the annual inflation rate between 2003 and 2009.

The results of the quantitative and qualitative analyses indicate that East Germany is more heavily affected by the introduction of the minimum wage than West Germany and Berlin. The qualitative analyses confirmed that the wages in West Germany and Berlin were usually, even before the introduction of the minimum wage, considerably higher than the introduced minimum wage level. This is considered to be the main reason for the observed differences in the level of impact.

We use two different treatment variables to measure the effect of the minimum wage on the growth of wages and employment. The first variable indicates the financial impact of the minimum wage introduction, because it measures how much the aggregate wages have to increase so that all employees are getting paid at least the minimum wage. The second treatment variable represents the share of employees who have received less than the minimum wage before it was introduced. This can be described as the personal impact of the minimum wage introduction on the facilities. 
The results of the estimations show a significant positive effect of the minimum wage on wage growth. This applies to the financial as well as to the personal impact. The comparative analyses indicate that the effects are at least partially attributable to a development independent of the minimum wage introduction. However, the analyses suggest that the introduction of the minimum wage had a positive effect on wage growth in East German institutions. This is confirmed by the qualitative studies.

Contrary to the results for the effects on wage growth, no significant effects of the treatment variables on employment growth can be found. The comparative analyses identify a significantly negative effect of the financial impact on employment growth. Analyses of the care statistics show a very strong fluctuation of the workforce in the care sector. The significantly negative results of the comparative analyses could indicate a larger staff fluctuation in facilities with low wages. This would affect the treatment variable and bias the estimation results.

In the case studies and expert interviews, an influence of the minimum wage on employment has been doubted. There is a shortage of skilled workers in the sector. Regardless of the minimum wage, employment growth is expected as a result of the increase in demand for nursing services due to the demographic change.

The impact of the minimum wage on competition is ambivalently perceived. Especially smaller facilities in East Germany seem to be affected by the minimum wage introduction and have lost their competitiveness against larger competitors. At the same time, the demand from the facilities that already paid wages above the minimum wage prior to its introduction is an indication that the minimum wage is partly used as a strategy of raising rivals' costs.

Most interviewees in the qualitative analyses welcomed the minimum wage introduction. Some criticised the different wage levels for East and West Germany and the lack of differentiation according to qualification levels.

Danksagung Wir danken den Forschungsdatenzentren des Instituts für Arbeitsmarkt- und Berufsforschung (IAB) sowie des statistischen Landesamtes Baden-Württemberg für die Bereitstellung der Daten. Ebenfalls möchten wir den befragten Einrichtungen sowie den Experten und Expertinnen für die umfassenden Hintergrundinformationen und für ihre Einschätzungen unseren Dank aussprechen. Insbesondere danken wir Bernhard Boockmann, Tobias Brändle, Günther Klee, Andreas Koch, Raimund Krumm, Michael Neumann, Pia Rattenhuber und Jochen Späth für wertvolle Anmerkungen sowie einem engagierten Team studentischer Hilfskräfte für ihre Unterstützung.

\section{Literatur}

Afentakis, A., Maier, T.: Projektionen des Personalbedarfs und -angebots in Pflegeberufen bis 2025. Wirtsch. Stat. 11, 990-1002 (2010)

Apel, H.: Das methodische Konzept der Fallstudien des ISG. ISG working paper Nr. 6, Institut für Sozialforschung und Gesellschaftspolitik (2009)
BA: Kennzahlen für interregionale Vergleiche im Rechtskreis SGB II. Bundesagentur für Arbeit, Nürnberg. Stand: 19.02.2010 (2010)

BMAS: Einigung der Pflegekommission auf einen Mindestlohn. Bundesministerium für Arbeit und Soziales, Berlin (2010)

Card, D., Krueger, A.: Minimum wages and employment: a case study of the fast-food industry in New Jersey and Pennsylvania. Am. Econ. Rev. 84(4), 772-793 (1994)

IAW: Evaluation bestehender Mindestlohnregelungen, Branche: Pflege. Abschlussbericht an das Bundesministerium für Arbeit, Tübingen (2011)

Kalina, T., Weinkopf, C.: Niedriglohnbeschäftigung 2008: Stagnation auf hohem Niveau - Lohnspektrum franst nach unten aus. IAQReport 2010-06, Universität Duisburg-Essen (2010)

Lamnek, S.: Qualitative Sozialforschung. Beltz Psychologie Verlags Union, Weinheim (2005)

Lührs, H.: die Zukunft der Arbeitsrechtlichen Kommissionen - Arbeitsbeziehungen in Den Kirchen und Ihren Wohlfahrtsverbänden Diakonie und Caritas Zwischen Kontinuität, Wandel und Umbruch. Wirtschafts- und Sozialpolitik. Nomos-Verlag, Baden Baden (2010)

Machin, S., Manning, A., Rahman, L.: Where the minimum wage bites hard: the introduction of the UK national minimum wage of a low wage sector. J. Eur. Econ. Assoc. 1(1), 154-180 (2003)

Rattenhuber, P.: Building the minimum wage: Germany's first sectorial minimum wage and its impact on wages in the construction industry. Discussion papers DIW Berlin 1111. Berlin (2011)

RWI; ADMED GmbH und Institute for Health Care Business GmbH: Auswirkungen von Mindestlöhnen auf Pflegeeinrichtungen und Pflegebedürftige. Projektbericht (2009)

Statistische Ämter des Bundes und der Länder: Regionalstatistik - Bevölkerungsstand, Wanderungen (2011)

Statistik der Bundesagentur für Arbeit: Arbeitsmarkt in Zahlen, Sozialversicherungspflichtig Beschäftigte nach Wirtschaftszweigen (WZ 2008) - Stand September 2011. Nürnberg, März (2012)

Statistisches Bundesamt: Qualitätsbericht: Statistik über ambulante Pflegeeinrichtungen (Pflegedienste) und Statistik über stationäre Pflegeeinrichtungen (Pflegeheime). Wiesbaden (2007)

T.I.P. Biehl \& Partner: Evaluation bestehender gesetzlicher Mindestlohnregelungen - Befragung der Pflegebranche in zwei Wellen, Ergebnisse der 1. Welle, September/Oktober (2010)

T.I.P. Biehl \& Partner: Evaluation bestehender gesetzlicher Mindestlohnregelungen - Befragung der Pflegebranche in zwei Wellen, Ergebnisse der 2. Welle, März/April (2011)

Katrin Harsch Studium von Grund- und Hauptschullehramt mit Schwerpunkt Grundschullehramt an der Pädagogischen Hochschule Weingarten (2004, Erste Staatsprüfung) sowie Soziologie, Betriebswirtschaftslehre und Ethnologie mit den Schwerpunkten empirische Sozialforschung, Marketing und Personalpolitik an der EberhardKarls-Universität Tübingen (2010, Magister Artium). Seit 2011 Wissenschaftliche Mitarbeiterin am Institut für Angewandte Wirtschaftsforschung e.V. (IAW) in Tübingen. Zu ihrem Tätigkeitsbereich zählen insbesondere qualitative und deskriptive Analysen sowie die Entwicklung und Durchführung standardisierter Befragungen in den Bereichen Arbeitsmarkt- und Sozialpolitik.

Hans Verbeek Studium der Volkswirtschaftslehre in Mannheim mit den Schwerpunkten Arbeitsmarktökonomik, Außenwirtschaft und Ökonometrie, 2009 Abschluss des Studiums als Diplom-Volkswirt. Seit 2009 ist er wissenschaftlicher Mitarbeiter am Institut für Angewandte Wirtschaftsforschung e.V. in Tübingen. Seine Forschungsschwerpunkte sind in der Evaluation arbeitsmarktpolitischer Maßnahmen sowie der Situation benachteiligter Jugendlicher und junger Erwachsener auf dem Arbeitsmarkt. 